Louisiana State University

LSU Digital Commons

Faculty Publications

Department of Physics \& Astronomy

$1-1-2012$

\title{
Late-time light curves of type II supernovae: Physical properties of supernovae and their environment
}

\author{
Masaaki Otsuka \\ Space Telescope Science Institute \\ Margaret Meixner \\ Space Telescope Science Institute \\ Nino Panagia \\ Space Telescope Science Institute \\ Joanna Fabbri \\ University College London \\ Michael J. Barlow \\ University College London
}

See next page for additional authors

Follow this and additional works at: https://digitalcommons.Isu.edu/physics_astronomy_pubs

\section{Recommended Citation}

Otsuka, M., Meixner, M., Panagia, N., Fabbri, J., Barlow, M., Clayton, G., Gallagher, J., Sugerman, B., Wesson, R., Andrews, J., Ercolano, B., \& Welch, D. (2012). Late-time light curves of type II supernovae: Physical properties of supernovae and their environment. Astrophysical Journal, 744 (1) https://doi.org/10.1088/ 0004-637X/744/1/26

This Article is brought to you for free and open access by the Department of Physics \& Astronomy at LSU Digital Commons. It has been accepted for inclusion in Faculty Publications by an authorized administrator of LSU Digital Commons. For more information, please contact ir@lsu.edu. 


\section{Authors}

Masaaki Otsuka, Margaret Meixner, Nino Panagia, Joanna Fabbri, Michael J. Barlow, Geoffrey C. Clayton, Joseph S. Gallagher, Ben E.K. Sugerman, Roger Wesson, Jennifer E. Andrews, Barbara Ercolano, and Douglas Welch 


\title{
LATE-TIME LIGHT CURVES OF TYPE II SUPERNOVAE: PHYSICAL PROPERTIES OF SUPERNOVAE AND THEIR ENVIRONMENT
}

\author{
Masaaki Otsuka $^{1,8}$, Margaret Meixner $^{1}$, Nino Panagia ${ }^{1}$, Johnna Fabbri $^{2}$, Michael J. Barlow ${ }^{2}$, \\ Geoffrey C. Clayton ${ }^{3}$, Joseph S. Gallagher ${ }^{4}$, Ben E. K. Sugerman ${ }^{5}$, Roger Wesson ${ }^{2}$, Jennifer E. Andrews ${ }^{3}$, \\ Barbara Ercolano ${ }^{6}$, ANd Douglas $\mathrm{Welch}^{7}$ \\ ${ }^{1}$ Space Telescope Science Institute, 3700 San Martin Dr., Baltimore, MD 21218, USA; otsuka@ stsci.edu \\ ${ }^{2}$ Department of Physics and Astronomy, University College London, Gower Street, London WC1E 6BT, UK \\ ${ }^{3}$ Department of Physics and Astronomy, Louisiana State University, Baton Rouge, LA 70803, USA \\ ${ }^{4}$ Department of Mathematics, Physics, and Computer Science, Raymond Walters College, 9555 Plain field Rd., Blue Ash, OH 45236, USA \\ ${ }^{5}$ Department of Physics and Astronomy, Goucher College, 1021 Dulaney Valley Road, Baltimore, MD 21204, USA \\ ${ }^{6}$ Universitäts-Sternwarte München, Scheinerstr. 1, 81679 München, Germany \\ ${ }^{7}$ Department of Physics and Astronomy, McMaster University, Hamilton, Ontario L8S 4M1, Canada \\ Received 2011 May 31; accepted 2011 August 23; published 2011 December 9
}

\begin{abstract}
We present BVRIJHK-band photometry of six core-collapse supernovae, SNe 1999bw, 2002hh, 2003gd, 2004et, $2005 \mathrm{cs}$, and 2006bc, measured at late epochs (>2 yr) based on the Hubble Space Telescope (HST), and the Gemini North, and WIYN telescopes. We also show the JHK light curves of supernova impostor SN 2008S up to day 575 because it was serendipitously in our SN 2002hh field of view. Of our 43 HST observations in total, 36 observations are successful in detecting the light from the SNe alone and measuring magnitudes of all the targets. HST observations show a resolved scattered light echo around SN 2003gd at day 1520 and around SN 2002hh at day 1717. Our Gemini and WIYN observations detected SNe 2002hh and 2004et as well. Combining our data with previously published data, we show VRIJHK-band light curves and estimate decline magnitude rates at each band in four different phases. Our prior work on these light curves and other data indicate that dust is forming in our targets from days $\sim 300$ to 400 , supporting SN dust formation theory. In this paper we focus on other physical properties derived from late-time light curves. We estimate ${ }^{56} \mathrm{Ni}$ masses for our targets $\left(0.5-14 \times 10^{-2} M_{\odot}\right)$ from the bolometric light curve of each of days $\sim 150-300$ using SN 1987A as a standard $\left(7.5 \times 10^{-2} M_{\odot}\right)$. The flattening or sometimes increasing fluxes in the late-time light curves of SNe 2002hh, 2003gd, 2004et, and 2006bc indicate the presence of light echoes. We estimate the circumstellar hydrogen density of the material causing the light echo and find that SN 2002hh is surrounded by relatively dense materials $\left(n(\mathrm{H})>400 \mathrm{~cm}^{-3}\right)$ and SNe 2003gd and 2004et have densities more typical of the interstellar medium $\left(\sim 1 \mathrm{~cm}^{-3}\right)$. We analyze the sample as a whole in the context of physical properties derived in prior work. The ${ }^{56} \mathrm{Ni}$ mass appears well correlated with progenitor mass with a slope of $0.31 \times 10^{-2}$, supporting the previous work by Maeda et al., who focus on more massive Type II SNe. The dust mass does not appear to be correlated with progenitor mass.
\end{abstract}

Key words: dust, extinction - supernovae: individual (SN1999bw, SN2002hh, SN 2003gd, SN2004et, SN2005cs, SN2006bc, SN 2)

\section{INTRODUCTION}

Since the discovery of SN 1987A, dust formation has been confirmed observationally in the ejecta of several core-collapse supernovae (Type II SNe; e.g., Wooden et al. 1993; Kozasa et al. 2009). In these, one or more of the following signatures are observed $\sim 250-600$ days after the initial explosion: (1) the development of an infrared excess seen in the spectral energy distribution (SED) of the SN; (2) the appearance of blueshifted optical/near-infrared lines, which is interpreted as the attenuation of the redshifted wing (i.e., the receding gas) by dust formed homogeneously in the ejecta; and (3) the steepening decline of the optical light curve consistent with an increase in circumstellar extinction. The SN luminosity after day $\sim 150$ is supported by $\gamma$ rays from ${ }^{56} \mathrm{Co}$ decaying into ${ }^{56} \mathrm{Ni}$. If the dust is formed in $\mathrm{SNe}$, the optical luminosity from the $\mathrm{SN}$ remnant has become much fainter than would be produced by the radioactive decay power.

In order to properly assess dust formation by Type II SNe ejecta it is essential to continue monitoring Type II SNe before

\footnotetext{
8 Current address: Institute of Astronomy and Astrophysics, Academia Sinica
} P.O. Box 23-141, Taipei 10617, Taiwan, R.O.C.; otsuka@ asiaa.sinica.edu.tw. and after dust formation started (i.e., day 300) because other physical properties such as the ${ }^{56} \mathrm{Ni}$ mass and light echoes from circumstellar and interstellar dust can be measured and disentangled from dust production effects. The early-epoch evolution of Type II SNe before day $\sim 300$ has been relatively well studied in the optical and near-IR bands. However at the present time, the evolution beyond day 300 has been poorly measured.

To address these issues, we have conducted long term photometric monitoring of $\mathrm{SNe}$ using high angular resolution facilities such as $H S T$ /WFPC2/NICMOS, WIYN/WHIRC, and Gemini/NIRI through our SNe project SEEDS (Survey for Evolution of Emission from Dust in SNe, PI: M. Barlow). In this paper we report on the late-epoch light curves of six Type II SNe. The remainder of this paper is organized as follows. In Section 2, we present the observations, data processing and resulting images, and light curves. In Section 3, we analyze the resulting light curves to derive ${ }^{56} \mathrm{Ni}$ masses for all targets except for SN 1999bw and, for cases with detected light echoes, to estimate the density of the environment. In Section 4, we discuss the results for each SN. In Section 5, we do a comparative analysis of these $\mathrm{SNe}$ in the context of physical properties 
reported in the literature, such as the amount of dust produced and progenitor mass.

\section{OBSERVATIONS AND DATA REDUCTION}

\subsection{Sample Selection}

We chose six Type II SNe in nearby galaxies, matching the following three criteria. (1) The distance to an SN is within $\sim 15 \mathrm{Mpc}$ and thus suitable for long-term follow-up observations with HST, Spitzer, and $>4$ m class ground-based telescopes. (2) At the start of the observations, the $\mathrm{SN}$ is 1 year or older after the initial explosion. (3) The expected $V$-band magnitude of targets is $<28$ based on the evolution of SN 1987A in order for the SNe to be detectable within a reasonable integration time. These six Type II SNe include four Type II-P (SNe 2002hh, 2003gd, 2004et, and 2005cs), one Type II-n (SN 1999bw), and one Type II-L or II-P (SN 2006bc). In addition, during our campaign, a supernova impostor, SN 2008S, erupted in the field of view of our SN 2002hh observations, allowing us to monitor this new type of variable as well.

\subsection{HST Observations}

The high-angular resolution images of our target SNe were taken by the HST with the Wide Field Planetary Camera 2 (WFPC2) and the Near Infrared Camera and Multi-Object Spectrometer 2 (NICMOS2) through the cycle 16 proposal by M. Meixner (proposal ID: GO 11229). The observations were coordinated so that the WFPC2 and NICMOS images were taken within days to a week of each other. In the WFPC2 observations, we took the images using the F450W, F606W, F622W, and F814W filters. In the NICMOS2 observations, we took the images using the F110W, F160W, and F205W filters. The observation logs and SN positions are summarized in Table 1. The boldface coordinates in this table are derived from our F606W or F622W HST observations and the others are from the SIMBAD database.

\subsection{HST Data Reduction}

The data reduction and calibration were performed by standard techniques. Using the STSDAS package version 3.8 specialized for $H S T$, we reduced and calibrated the data, including cosmic rays and artificial features removal and detector linearity correction. The final high resolution images were created using the STSDAS/DRIZZLE package. For both the WFPC2 and NICMOS2 observations, we conducted sub pixel scale dithering to improve signal-to-noise ratio and remove cosmic rays and obtain further high-resolution images via the drizzle technique. We set four or five points dithering with 115-400 s per science frame for each band. After DRIZZLE processing, we performed an additional distortion correction and alignment using background stars for the NICMOS2 data. The point-spread function (PSF) of these reference background stars were fit by Gaussian profiles to obtain accurate positions, and then instrumental distortions were corrected with the XYXYMATCH, GEOMAP, and GEOTRAN IRAF routines, and we aligned the positions of stars in the NICMOS detector to those in the WFPC2. The resultant plate scale reaches 0.025 pixel $^{-1}$ in the WFPC2 and archived ACS/HRC data (see below) and $0 . ' 04$ pixel $^{-1}$ in the NICMOS2 and archival NICMOS3 data (see below), respectively. The size of the PSF at FWHM typically corresponds to $\sim 3$ pixel. Using the PSF fitting package IRAF/DAOPHOT, photometry was performed on the images, except for SN 2005cs in F110W and
F160W and SN 2002hh in all bands. For SN 2005cs in F110W and F160W, we used differential images with pre-explosion images using archival NICMOS3 images of the host galaxy taken in 1998 June 28 (PI: N. Scoville, Proposal-ID: GO 7237) and our NICMOS2 data to detect the SN alone and performed PSF fitted photometry. SN 2002hh has an extended shell structure as shown in Figure 1; we therefore performed aperture photometry based on background star subtracted images. We used 0.'6 radius regions to define the entire the $\mathrm{SN}$. We subtracted the background from an annulus centered on the $\mathrm{SN}$ with inner and outer radii of $00^{\prime} 6$ and $0 . ' 8$. The aperture corrections were calculated with an empirical PSF function. Note that the magnitudes of SN2002hh listed in Tables 1-3 include the SN + extend light echo.

For each filter, the measured count rate $(\mathrm{CR}$, in units of $\mathrm{DN} \mathrm{s}^{-1}$ ) of the $\mathrm{SN}$ was converted to flux by multiplication with the PHOTFLAM (erg s${ }^{-1} \mathrm{~cm}^{-2} \AA^{-1} \mathrm{DN}^{-1}$ or erg s $\mathrm{sm}^{-1} \mathrm{~cm}^{-2}$ $\AA^{-1}$ electron $^{-1}$ ) conversion factor given in the fits header, where PHOTFLAM is the bandpass-averaged flux density for a source that would produce a count rate (CR in units of $D N$ $\mathrm{s}^{-1}$ or electron $\mathrm{s}^{-1}$ ). The HST system magnitude (STMAG) was calculated from the following equation:

$$
\text { STMAG }=-2.5 \log _{10}(\mathrm{CR} \times \text { PHOTFLAM })+\mathrm{ZP},
$$

where $\mathrm{ZP}$ is the zero-point magnitude for $1 \mathrm{DN} \mathrm{s}^{-1}$ or 1 electron $\mathrm{s}^{-1}$. For the WFPC2 and archival ACS data (see below), the conversion from F450W, F606W and F622W, and F814W STMAG to the Vega magnitude system $B, V, R$, and $I$, respectively, used the STSDAS/SYNPHOT package. The broadband NICMOS filters, F110W, F160W, and F205W, are roughly equivalent to the $J, H$, and $K$ filters, respectively; color transformations are not well constrained for late-time SN spectra at near-IR wavelengths. Based on our experience, for SNe in days 300-1000, the SED in the range from $\sim 0.44$ to $\sim 2.2 \mu \mathrm{m}$ can be represented by a single blackbody function with the effective temperature of $\sim 7000-11,000 \mathrm{~K}$. The conversion from the STMAG to the Vega magnitude system was done using the STSDAS/SYNPHOT package, assuming a $9000 \mathrm{~K}$ blackbody function as the incident SED.

\subsection{HST Archive Data}

Except for SN 2004et, archival WFPC2, Advanced Camera for Surveys (ACS), and NICMOS3 images are available for our SNe sample as noted in Table 2. We created drizzled images and we measured the magnitudes by the same way as described above. The measured magnitudes are listed in Table 2. In this table we also list the magnitudes measured at earlier epochs as found in the literature as noted in the table.

\subsection{NIRI and WHIRC Observations}

To complement HST observations, we performed $J H K$-band high-resolution imaging observations for SNe 2002hh, 2004et, and 2008S using the Gemini North $8.2 \mathrm{~m}$ telescope with the Near Infrared Imager and spectrometer (NIRI) and the WIYN $3.5 \mathrm{~m}$ telescope with the WIYN High Resolution Infrared Camera (WHIRC; Meixner et al. 2010). The NIRI and 2009 August WHIRC observations were carried out through the NOAO open use programs of PIs G. C. Clayton and M. Otsuka, respectively. The other WHIRC observations were carried out in STScI guaranteed observation time (PI: M. Meixner). The plate scale of both instruments is $\sim 0^{\prime} .1$ pixel $^{-1}$ and the seeing was 0 '.3-0!.8 at $J H K$-bands. The average PSF size of $\sim 0.5$ enabled 

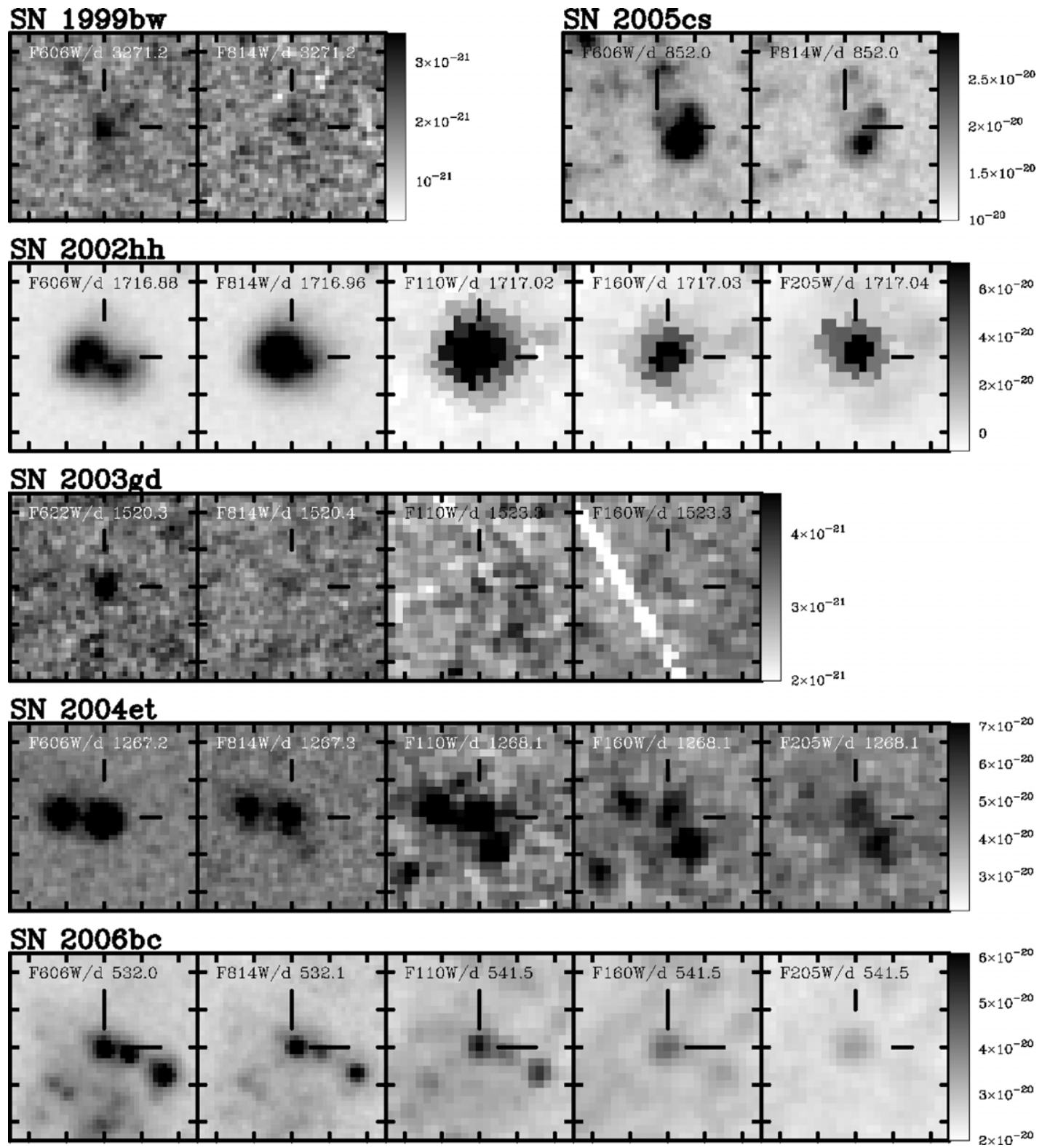

Figure 1. HST images of SNe 1999bw, 2002hh, 2003gd, 2004et, 2005cs, and 2006bc. The SN is in the center of each panel. The size of each panel is $1^{\prime \prime} \times 1^{\prime \prime}$ and each tick mark is $0^{\prime}$. 2. The intensity scale ( $\mathrm{erg} \mathrm{s}^{-1} \mathrm{~cm}^{-2} \AA^{-1}$ pixel $^{-1}$ ) is common in each SN. In all cases north is up and east is to the left. Each box has a label for $H S T$ filter and day after explosion.

us to measure magnitudes of both $\mathrm{SNe}$ with relatively little contamination from nearby stars.

The data reductions using the IRAF/MSCRED package were carried out in a standard manner (dark subtraction, flat-fielding, and local sky subtraction). For the WHIRC data, we performed a detector linearity correction using the WHIRC task WPREP and distortion correction using the files downloaded from the WIYN/WHIRC Web page. The magnitude measurements were performed using IRAF/DAOPHOT. The conversion of the instrumental magnitudes into the standard system was performed relative to Two Micron All-Sky Survey (2MASS) photometry of 4-10 stars in the field.

The measured magnitudes are listed in Table 3. The errors include the estimated errors of 2MASS stars' magnitudes. For SN 2002hh, we converted the Vega magnitudes derived from HST data to 2MASS magnitudes. Comparison between HST and 2MASS magnitudes indicates that a star near SN 2002hh contributes $\sim 0.2-0.3 \mathrm{mag}$.

\subsection{Results}

The resultant images of target $\mathrm{SNe}$ are presented in Figure 1. We are successful in detecting the $\mathrm{SNe}$ and measuring either $\mathrm{F} 606 \mathrm{~W}(V)$ - or $\mathrm{F} 622 \mathrm{~W}(R)$-band magnitudes of all the targets in $H S T$ observations. The light curves of each SN are presented in Figures 2 (VRI) and 3 ( $J H K$-bands). These light curves are based on our data (filled circles) and data from the literature for each object (open circles). The data after $>600-1000$ days are mainly from the former and the data before this phase are mainly from the latter. The data sources for each object are summarized in Table 4. The values in Figures 2 and 3 are de-reddened absolute magnitudes adopting the distance to the host galaxy, $D$, and $E(B-V)$ listed in the third and fourth columns of Table 1. All $E(B-V)$ in Table 1 are the total extinction by the Milky Way and host galaxy. We used the reddening function assuming the $R_{V}=3.1$ case by Cardelli et al. (1989) for all SNe. As references, we plot the light curves of the Type II-P SNe 1987A 
Table 1

HST Observation Log and Measured Magnitudes

\begin{tabular}{|c|c|c|c|c|c|c|c|c|c|c|c|c|}
\hline SN Name & Host Galaxy & $D(\mathrm{Mpc})$ & $E(B-V)$ & $\alpha(2000.0)$ & $\delta(2000.0)$ & Obs. Date & Epoch (day) & Exp. Time & Detector & Filter & Detect & Magnitudes \\
\hline$\overline{\text { SN1999bw }}$ & NGC 3198 & 13.7 & 0.013 & 10:19:46.81 & $+45: 31: 35.0$ & 2008 Apr 3 & 3271.24 & $128 \mathrm{~s} \times 5$ & NIC2 & F110W & $\mathrm{N}$ & $\ldots$ \\
\hline \multirow[t]{4}{*}{ (II-n) } & & & & $10: 19: 46.68$ & $+45: 31: 37.32$ & 2008 Apr 3 & 3271.25 & $128 \mathrm{~s} \times 4$ & NIC2 & F160W & $\mathrm{N}$ & $\ldots$ \\
\hline & & & & & & 2008 Apr 3 & 3271.26 & $115 \mathrm{~s} \times 5$ & NIC2 & F205W & $\mathrm{N}$ & \\
\hline & & & & & & 2008 Apr 3 & 3271.11 & $400 \mathrm{~s} \times 4$ & $\mathrm{PC} 1$ & F606W & Y & $25.77 \pm 0.16$ \\
\hline & & & & & & 2008 Apr 3 & 3271.20 & $400 \mathrm{~s} \times 4$ & PC1 & F814W & $\mathrm{Y}$ & $25.55 \pm 0.24$ \\
\hline \multirow{6}{*}{$\begin{array}{l}\text { SN2002hh } \\
\text { (II-P) }\end{array}$} & NGC 6946 & 5.9 & 1.97 & $20: 34: 44.29$ & $+60: 07: 19.0$ & $2007 \mathrm{Jul} 9$ & 1716.88 & $400 \mathrm{~s} \times 4$ & PC1 & F606W & $\mathrm{Y}$ & $20.62 \pm 0.11$ \\
\hline & & & & 20:34:44.19 & $+60: 07: 20.11$ & $2007 \mathrm{Jul} 9$ & 1716.91 & $400 \mathrm{~s} \times 4$ & PC1 & F814W & $\mathrm{Y}$ & $19.67 \pm 0.11$ \\
\hline & & & & & & 2007 Jul 9 & 1716.96 & $400 \mathrm{~s} \times 4$ & PC1 & F450W & $\mathrm{Y}$ & $23.25 \pm 0.18$ \\
\hline & & & & & & $2007 \mathrm{Jul} 10$ & 1717.02 & $128 \mathrm{~s} \times 5$ & NIC2 & F110W & $\mathrm{Y}$ & $18.84 \pm 0.11$ \\
\hline & & & & & & $2007 \mathrm{Jul} 10$ & 1717.03 & $128 \mathrm{~s} \times 4$ & NIC2 & F160W & $\mathrm{Y}$ & $18.53 \pm 0.11$ \\
\hline & & & & & & $2007 \mathrm{Jul} 10$ & 1717.04 & $115 \mathrm{~s} \times 5$ & NIC2 & F205W & $\mathrm{Y}$ & $17.44 \pm 0.11$ \\
\hline \multirow{5}{*}{$\begin{array}{l}\text { SN2003gd } \\
\text { (II-P) }\end{array}$} & NGC 628 & 7.2 & 0.18 & $01: 36: 42.65$ & $+15: 44: 19.9$ & 2007 Aug 11 & 1519.79 & $400 \mathrm{~s} \times 4$ & PC1 & F622W & $\mathrm{Y}$ & $24.91 \pm 0.18$ \\
\hline & & & & 01:36:42.71 & $+15: 44: 20.25$ & 2007 Aug 11 & 1519.86 & $400 \mathrm{~s} \times 4$ & PC1 & F814W & $\mathrm{Y}$ & $25.60 \pm 0.19$ \\
\hline & & & & & & 2007 Aug 14 & 1523.25 & $128 \mathrm{~s} \times 5$ & NIC2 & F110W & $\mathrm{Y}$ & $<24.92$ \\
\hline & & & & & & 2007 Aug 14 & 1523.26 & $128 \mathrm{~s} \times 4$ & NIC2 & F160W & $\mathrm{Y}$ & $<25.47$ \\
\hline & & & & & & 2007 Aug 14 & 1523.27 & $115 \mathrm{~s} \times 5$ & NIC2 & F205W & $\mathrm{N}$ & $\ldots$ \\
\hline \multirow{10}{*}{$\begin{array}{l}\text { SN2004et } \\
\text { (II-P) }\end{array}$} & NGC 6946 & 5.9 & 0.41 & $20: 35: 25.33$ & $+60: 07: 17.7$ & 2007 Jul 8 & 1071.74 & $400 \mathrm{~s} \times 4$ & $\mathrm{PC} 1$ & F606W & Y & $23.01 \pm 0.12$ \\
\hline & & & & $20: 35: 25.36$ & $+60: 07: 17.80$ & $2007 \mathrm{Jul} 8$ & 1071.82 & $400 \mathrm{~s} \times 4$ & PC1 & F814W & $\mathrm{Y}$ & $23.04 \pm 0.14$ \\
\hline & & & & & & $2007 \mathrm{Jul} 8$ & 1071.91 & $128 \mathrm{~s} \times 5$ & NIC2 & F110W & $\mathrm{Y}$ & $21.76 \pm 0.14$ \\
\hline & & & & & & $2007 \mathrm{Jul} 8$ & 1071.94 & $128 \mathrm{~s} \times 4$ & NIC2 & F160W & $\mathrm{Y}$ & $22.56 \pm 0.22$ \\
\hline & & & & & & $2007 \mathrm{Jul} 8$ & 1071.95 & $115 \mathrm{~s} \times 5$ & NIC2 & F205W & Y & $<22.07$ \\
\hline & & & & & & 2008 Jan 19 & 1266.71 & $400 \mathrm{~s} \times 4$ & PC1 & F606W & $\mathrm{Y}$ & $23.35 \pm 0.11$ \\
\hline & & & & & & 2008 Jan 19 & 1266.78 & $400 \mathrm{~s} \times 4$ & PC1 & F814W & $\mathrm{Y}$ & $23.35 \pm 0.13$ \\
\hline & & & & & & 2008 Jan 20 & 1267.58 & $128 \mathrm{~s} \times 5$ & NIC2 & F110W & $\mathrm{Y}$ & $21.99 \pm 0.15$ \\
\hline & & & & & & 2008 Jan 20 & 1267.59 & $128 \mathrm{~s} \times 4$ & NIC2 & F160W & $\mathrm{Y}$ & $22.62 \pm 0.24$ \\
\hline & & & & & & 2008 Jan 20 & 1267.59 & $115 \mathrm{~s} \times 5$ & NIC2 & F205W & $\mathrm{Y}$ & $21.07 \pm 0.19$ \\
\hline \multirow{7}{*}{$\begin{array}{l}\text { SN2005cs } \\
\text { (II-P) }\end{array}$} & NGC 5194 & 7.1 & 0.14 & $13: 29: 53.37$ & $+47: 10: 28.2$ & 2007 Oct 28 & 851.96 & $400 \mathrm{~s} \times 4$ & $\mathrm{PC} 1$ & F606W & $\mathrm{Y}$ & $25.16 \pm 0.19$ \\
\hline & & & & $13: 29: 52.80$ & $+47: 10: 35.64$ & 2007 Oct 28 & 852.02 & $400 \mathrm{~s} \times 4$ & $\mathrm{PC} 1$ & F814W & $\mathrm{Y}$ & $25.07 \pm 0.24$ \\
\hline & & & & & & 2007 Oct 31 & 854.15 & $128 \mathrm{~s} \times 5$ & NIC2 & F110W & $\mathrm{Y}$ & $<\mathbf{2 3 . 3 0}$ \\
\hline & & & & & & 2007 Oct 31 & 854.16 & $128 \mathrm{~s} \times 4$ & NIC2 & F160W & $\mathrm{N}$ & $\ldots$ \\
\hline & & & & & & 2007 Oct 31 & 854.17 & $115 \mathrm{~s} \times 5$ & NIC2 & F205W & $\mathrm{N}$ & $\ldots$ \\
\hline & & & & & & 2008 Mar 31 & 1006.15 & $400 \mathrm{~s} \times 4$ & PC1 & F606W & Y & $<25.94$ \\
\hline & & & & & & 2008 Mar 31 & 1006.21 & $400 \mathrm{~s} \times 4$ & PC1 & F814W & $\mathrm{N}$ & \\
\hline SN2006bc & NGC 2397 & 16 & 0.52 & $07: 21: 16.50$ & $-68: 59: 57.3$ & 2007 Sep 7 & 531.98 & $400 \mathrm{~s} \times 4$ & $\mathrm{PC} 1$ & F606W & $\mathrm{Y}$ & $23.16 \pm 0.19$ \\
\hline \multirow[t]{9}{*}{ (II-P or L) } & & & & $07: 21: 16.45$ & $-68: 59: 56.75$ & 2007 Sep 7 & 532.07 & $400 \mathrm{~s} \times 4$ & $\mathrm{PC} 1$ & F814W & $\mathrm{Y}$ & $21.72 \pm 0.19$ \\
\hline & & & & & & 2007 Sep 17 & 541.45 & $128 \mathrm{~s} \times 5$ & NIC2 & F110W & $\mathrm{Y}$ & $21.60 \pm 0.20$ \\
\hline & & & & & & 2007 Sep 17 & 541.46 & $128 \mathrm{~s} \times 4$ & NIC2 & F160W & $\mathrm{Y}$ & $20.80 \pm 0.20$ \\
\hline & & & & & & 2007 Sep 17 & 541.47 & $115 \mathrm{~s} \times 5$ & NIC2 & F205W & $\mathrm{Y}$ & $19.90 \pm 0.10$ \\
\hline & & & & & & $2008 \mathrm{Feb} 17$ & 694.25 & $400 \mathrm{~s} \times 4$ & PC1 & F606W & $\mathrm{Y}$ & $23.85 \pm 0.26$ \\
\hline & & & & & & 2008 Feb 17 & 694.28 & $400 \mathrm{~s} \times 4$ & $\mathrm{PC} 1$ & F814W & $\mathrm{Y}$ & $22.51 \pm 0.31$ \\
\hline & & & & & & 2008 Feb 17 & 694.35 & $128 \mathrm{~s} \times 5$ & NIC2 & F110W & Y & $22.40 \pm 0.30$ \\
\hline & & & & & & 2008 Feb 17 & 694.36 & $128 \mathrm{~s} \times 4$ & NIC2 & F160W & $\mathrm{Y}$ & $21.70 \pm 0.40$ \\
\hline & & & & & & 2008 Feb 17 & 694.37 & $115 \mathrm{~s} \times 5$ & NIC2 & F205W & Y & $20.70 \pm 0.20$ \\
\hline
\end{tabular}

Notes. The measured magnitudes at F450W, F606W, F622W, F814W, F110W, F160W, and F205W in column (10) are converted to $B, V, R, I, J, H$, and $K$ magnitudes. We converted the STMAG into the Johnson-Cousins system and into the

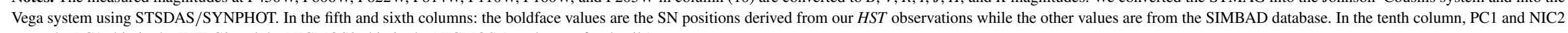
mean the PC1 chip in the WFPC2 and the NICMOS2 chip in the NICMOS (see the text for details).

References. (For $D$ and $E(B-V)$ ) SN 1999bw: Freedman et al. 2001, Smith et al. 2011; SN 2002hh: Barlow et al. 2005, Karachentsev et al. 2000, Pozzo et al. 2006; SN 2003gd: Van Dyk et al. 2003, Hendry et al. 2005; SN 2004et: Fabbri et al. 2011, Karachentsev et al. 2000; SN 2005cs: Takáts \& Vinkó 2006, Tsvetkov et al. 2006; SN 2006bc: Gallagher et al. 2010; Mihos \& Bothun 1997. 
Table 2

Magnitudes Obtained in Previous HST Observations

\begin{tabular}{|c|c|c|c|c|c|c|}
\hline SN Name & Obs. Date & Epoch & Detector $^{\mathrm{a}}$ & Filter & Magnitudes & Ref. \\
\hline SN 1999bw & $2001 \operatorname{Jan} 9$ & 629.88 & PC1 & $\mathrm{F} 555 \mathrm{~W}(V)$ & $24.08 \pm 0.06$ & $\overline{(1)}$ \\
\hline \multirow[t]{3}{*}{ SN 2002hh } & 2005 Sep 17 & 1056.26 & HRC & $\mathrm{F} 606 \mathrm{~W}(V)$ & $20.88 \pm 0.11$ & (2) \\
\hline & 2005 Sep 17 & 1056.40 & HRC & $\mathrm{F} 814 \mathrm{~W}(I)$ & $19.49 \pm 0.11$ & (2) \\
\hline & 2006 Apr 23 & 1273.94 & HRC & $\mathrm{F} 606 \mathrm{~W}(V)$ & $20.91 \pm 0.11$ & (2) \\
\hline \multirow[t]{4}{*}{ SN 2003gd } & 2004 Dec 8 & 544.11 & HRC & $\mathrm{F} 435 \mathrm{~W}(B)$ & $23.98 \pm 0.15$ & (3) \\
\hline & 2004 Dec 8 & 544.11 & HRC & $\mathrm{F} 622 \mathrm{~W}(R)$ & $23.13 \pm 0.16$ & (3) \\
\hline & 2007 Jun 21 & 1469.45 & PC1 & $\mathrm{F} 450 \mathrm{~W}(B)$ & $25.01 \pm 0.17$ & (4) \\
\hline & 2007 Jun 21 & 1469.45 & PC1 & $\mathrm{F} 675 \mathrm{~W}(R)$ & $24.10 \pm 0.16$ & (4) \\
\hline \multirow[t]{2}{*}{ SN 2005cs } & 2006 Dec 10 & 529.02 & HRC & $\mathrm{F} 555 \mathrm{~W}(V)$ & $22.07 \pm 0.15$ & (5) \\
\hline & 2006 Dec10 & 529.02 & HRC & $\mathrm{F} 814 \mathrm{~W}(I)$ & $21.36 \pm 0.15$ & (5) \\
\hline \multirow[t]{2}{*}{ SN 2006bc } & 2006 Oct 14 & 203.28 & WFC1 & $\mathrm{F} 555 \mathrm{~W}(V)$ & $22.10 \pm 0.10$ & (6) \\
\hline & 2006 Oct 14 & 203.28 & WFC1 & $\mathrm{F} 814 \mathrm{~W}(I)$ & $19.80 \pm 0.10$ & (6) \\
\hline
\end{tabular}

Note. ${ }^{\text {a }}$ HRC means High Resolution Camera mode in the ACS. WFC1 means the Wide Field Camera 1 chip in the WFPC2. References. (1) Li et al. 2002; (2) This work (data source; GO 10607, PI: Sugerman); (3) Sugerman 2005; (4) Maund \& Smartt 2009; (5) This work (data source; GO 10877, PI: Li); (6) Gallagher et al. (2011; GO 14980, PI: Smartt).

Table 3

NIRI and WHIRC Observations

\begin{tabular}{|c|c|c|c|c|c|}
\hline \multicolumn{6}{|c|}{ SN 2002hh } \\
\hline Obs. Date & Epoch & Instr. & $J$ & $H$ & $K$ \\
\hline 2005 Jun 4 & 951.5 & NIRI & $18.50 \pm 0.16$ & $17.76 \pm 0.16$ & $16.93 \pm 0.20$ \\
\hline 2005 Aug 4 & 1012.5 & NIRI & $18.57 \pm 0.15$ & $17.80 \pm 0.16$ & $16.93 \pm 0.20$ \\
\hline 2005 Oct 16 & 1085.3 & NIRI & $>18.48$ & $>17.88$ & $16.78 \pm 0.20$ \\
\hline 2006Jul 7 & 1349.5 & NIRI & $18.51 \pm 0.15$ & $17.75 \pm 0.16$ & $16.95 \pm 0.20$ \\
\hline 2007 Jul 10 & 1717.0 & $H S T$ & $18.61 \pm 0.19$ & $18.03 \pm 0.23$ & $17.02 \pm 0.21$ \\
\hline 2008 Nov 12 & 2208.1 & WHIRC & $18.59 \pm 0.15$ & $17.82 \pm 0.16$ & $17.00 \pm 0.20$ \\
\hline 2009 May 10 & 2387.4 & WHIRC & $\ldots$ & $17.54 \pm 0.20$ & $\ldots$ \\
\hline 2009 Aug 30 & 2499.3 & WHIRC & $\ldots$ & $18.07 \pm 0.20$ & $\ldots$ \\
\hline \multicolumn{6}{|c|}{ SN 2004et } \\
\hline 2009Aug 30 & 1801 & WHIRC & $\ldots$ & $<22.6$ & $\ldots$ \\
\hline \multicolumn{6}{|l|}{ SN 2008S } \\
\hline 2008 Nov 12 & 283.3 & WHIRC & $18.37 \pm 0.15$ & $16.73 \pm 0.15$ & $15.64 \pm 0.20$ \\
\hline 2009 May 10 & 463.5 & WHIRC & $\ldots$ & $17.83 \pm 0.16$ & $16.25 \pm 0.73$ \\
\hline 2009 Aug 30 & 575.5 & WHIRC & $\ldots$ & $18.74 \pm 0.16$ & $\ldots$ \\
\hline
\end{tabular}

Notes. The errors of 2MASS stars' magnitudes are included. In SN 2002hh, the HST magnitudes were recalibrated using the NIRI data and nearby 2MASS stars' magnitude.

(Hamuy \& Suntzeff 1990; Walker \& Suntzeff 1991) and 1999em (Elmhamdi et al. 2003), which are well-studied Type II SNe.

We measured the rate of fading of $\mathrm{SNe} \gamma_{\text {band }}$ during four phases, namely, days $\sim 30-100$ (plateau phase), $\sim 150-300$ (radioactive decay phase), $300-800$ (dust forming phase), and $>800$. The results are summarized in Table 5. For each phase, we fit a line to the light curve, the slope of which is the fading rate for that phase measured in units of magnitude per 100 days. The values in boldface are derived from the data presented in this work. We note that light echoes, which may appear as flattening or increasing fluxes in the light curve, could arise during any of these phases depending on the location of the scattering material with respect to the SNe. The light curves of Type II SNe during the radioactive decay phase can be explained by the $\gamma$-ray power of the radioactive decay of ${ }^{56} \mathrm{Co}$ to ${ }^{56} \mathrm{Fe}$, at a rate corresponding to the $e$-folding lifetime of the ${ }^{56} \mathrm{Co}$ decay $\left(t_{56}=111.3\right.$ days $)$. The rate expected for radioactive decay of ${ }^{56} \mathrm{Co}$ into ${ }^{56} \mathrm{Fe}$ is $0.98 \mathrm{mag}$ per 100 days, while the averaged $\gamma_{V}$ among eight SNe is $0.96 \pm 0.12$ (Patat et al. 1994). For SN 2004et, for example, Sahu et al. (2006) found that the $B V R I$ light curves in days 180-310 were linear with $\gamma_{V}=1.04$,
$\gamma_{R}=1.01, \gamma_{I}=1.07$. Maguire et al. (2010) also obtained similar results $(1.02,0.92$, and 1.09 , respectively). Our estimated $\gamma_{\text {VRI }}$ except for SNe 2002hh and 2005cs are in good agreement with Patat et al. (1994). Two SNe might have additional power sources in this phase (see Sections 4.2 for SN 2002hh and 4.5 for $\mathrm{SN} 2005 \mathrm{cs})$. We find that $\gamma_{J H K}$ are $\sim 1-1.4$ for all objects. Beyond $\sim 300$ days, the fading rates are steeper than the ${ }^{56}$ Co decay because the opacity to $\gamma$-rays is decreased and dust starts forming. This supports SN dust formation theory. We find flattening or increasing magnitude features in SNe 1999bw, 2002hh, 2003gd, 2004et, and 2006bc at the same epoch. We will discuss whether these SNe have light echoes based on the surrounding interstellar medium (ISM) density later.

\section{ANALYSIS OF LATE-TIME LIGHT CURVES}

In our prior work on the late-time light curves of these $\mathrm{SNe}$, we have concentrated on the dust formation process and estimated dust mass as noted in Section 4. In this paper, we analyze these light curves for two other quantities: the ejected ${ }^{56} \mathrm{Ni}$ mass (Table 6) and the density of the environment when a light echo 

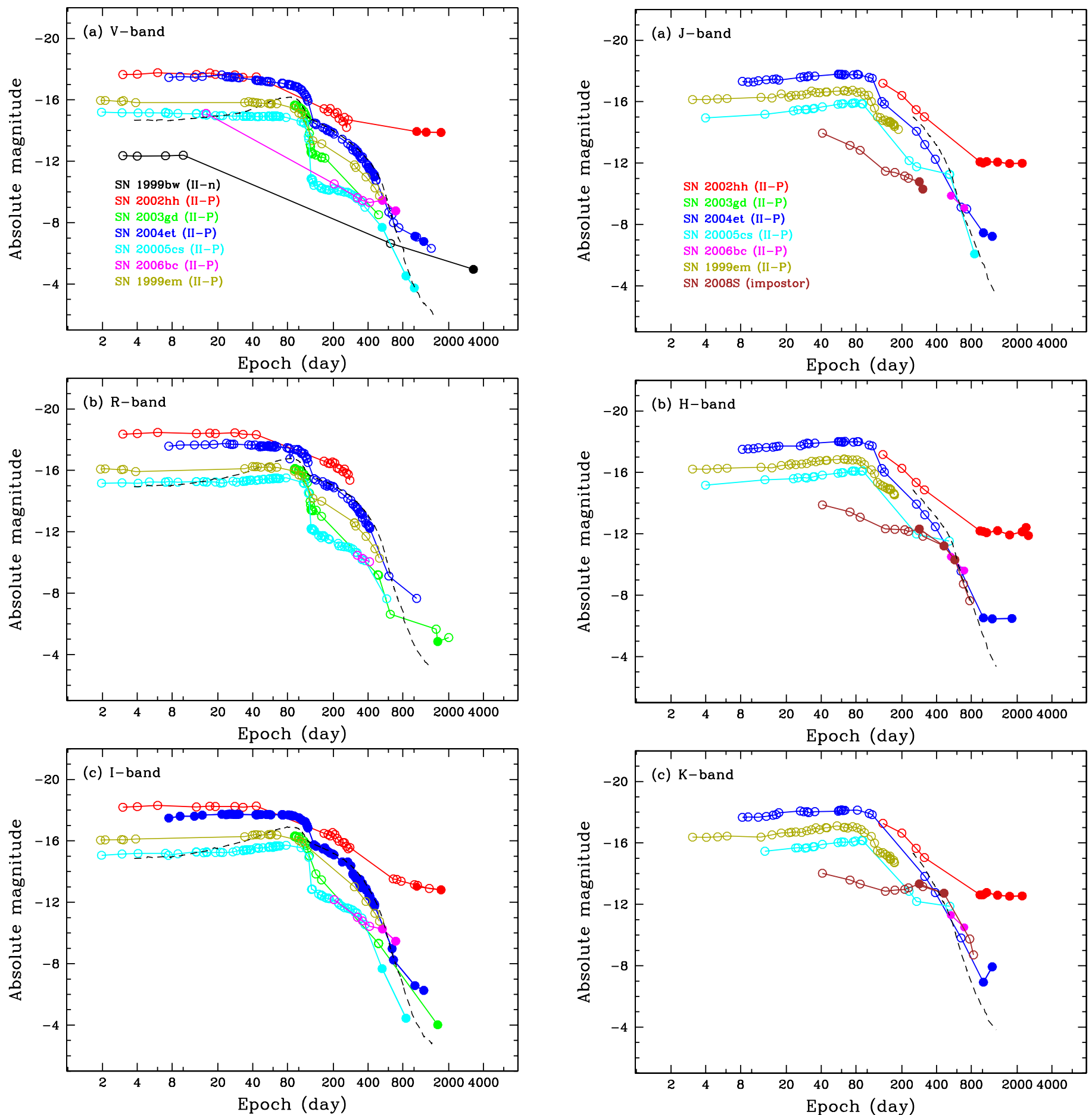

Figure 2. VRI light curves on a de-reddened absolute magnitude scale. The open circles are from published papers and the filled circles indicate our contributions. The broken lines are from published SN 1987A data. The data sources are listed in Table 4.

is present (Table 8 ). Table 8 summarizes the derived physical parameters for these $\mathrm{SNe}$ including ${ }^{56} \mathrm{Ni}$ mass, the environment density, dust produced and progenitor mass. Below we describe how we calculate the ${ }^{56} \mathrm{Ni}$ mass and the environment density.

\subsection{Mass of ${ }^{56} \mathrm{Ni}$}

Here, we focus on the ${ }^{56} \mathrm{Ni}$ mass synthesized in the $\mathrm{SN}$ explosion, because we can estimate this mass using bolometric light curves during radioactive decay.

Figure 3. Light curves at $J H K$-bands in de-reddened absolute magnitude scale. The symbols and lines are the same meanings in Figure 1. The data sources are listed in Table 4.

The method for ${ }^{56} \mathrm{Ni}$ mass estimation is to (1) determine the bolometric luminosity light curve for the $\mathrm{SNe}$ and (2) compare the slope during radioactive decay between targets and a standard SN. Here, we use SN 1987A as a standard. Arnett \& Fu (1989) estimated $0.073 \pm 0.015 M_{\odot}$ of freshly synthesized ${ }^{56} \mathrm{Ni}$ in $\mathrm{SN} 1987 \mathrm{~A}$ by comparing the observed bolometric luminosity curve and theoretical model.

By using simple blackbody fitting techniques, we estimated the luminosity $L_{\text {hot }}$ and emitting radius $R_{\text {hot }}$ of the hot component $T_{\text {eff }}$ (>5000 K here). We estimated these three quantities based 
Table 4

The Data Source of Type II SN and SN 2008S Light Curves

\begin{tabular}{ll}
\hline \hline SN Name & \multicolumn{1}{c}{ References } \\
\hline SN 1999bw & Li et al. (2002); Smith et al. (2011); This work \\
SN 2002hh & Pozzo et al. (2006); Welch et al. (2007); This work \\
SN 2003gd & Hendry et al. (2005); Maund \& Smartt (2009); This work \\
SN 2004et & Fabbri et al. (2011); Kotak et al. (2009); This work \\
SN 2005cs & Pastorello et al. (2009); Tsvetkov et al. (2006); This work \\
SN 2006bc & Brown et al. (2009); Gallagher et al. (2010); This work \\
SN 1987A & Hamuy \& Suntzeff (1990); Walker \& Suntzeff (1991) \\
SN 1999em & Elmhamdi et al. (2003) \\
\hline SN 2008S & Botticella et al. (2009); Prieto et al. (2010); This work
\end{tabular}

on BVRIJHK. Actually, there are archival $U V$ data only in the very early phase. For SN 2004et, Fabbri et al. (2011) performed multi-component blackbody fittings to the BVRIJHK and Spitzer IRAC four bands and MIPS $24 \mu \mathrm{m}$ data. They showed that the percentages of hot component to the total luminosity exceeded $76 \%$ from days 64 to 464 . We assume that the condition is the same as in the other $\mathrm{SNe}$, i.e., the hot component represented by optical- to near-IR SED is the main component of the bolometric luminosity. Since we are interested in the late-epoch SEDs, we selected days 150, 300, and 600. The flux densities at each band are estimated using the fading rate in Table 5. The results of SED fittings are summarized in Table 6. The uncertainties of $L_{\text {hot }}$ and $T_{\text {eff }}$ are $\sim 20 \%$ of the estimated values and $\pm \sim 500 \mathrm{~K}$.

Next, we estimated the ejected ${ }^{56} \mathrm{Ni}$ mass based on the assumption that the energy source of our sample $\mathrm{SNe}$ beyond 150 days is mainly from the power of gamma rays emitted through ${ }^{56} \mathrm{Co}$ to ${ }^{56} \mathrm{Fe}$ radioactive decay. As the ejecta expand, their opacity to $\gamma$-rays is expected to decrease, which results in a modified light curve given by Woosley et al. (1989):

$L_{\text {total }}=$ Const $\times \exp \left(-\frac{t}{t_{56}}\right)\left[1-\exp \left(-\kappa_{56, \gamma} \phi_{0}\left(\frac{t_{0}}{t}\right)^{2}\right)\right]$,

where $t_{56}$ is the $e$-folding lifetime of ${ }^{56} \mathrm{Co}$ and the term in the brackets is the effective opacity; $\kappa_{56, \gamma}=0.033 \mathrm{~cm}^{2} \mathrm{~g}^{-1}$ is the average opacity to ${ }^{56} \mathrm{Co}$-decay $\gamma$-rays, and $\phi_{0}=7 \times$ $10^{4} \mathrm{~g} \mathrm{~cm}^{-2}$ is the column depth at the fiducial time $t_{0}=$ 11.6 days. Fitting Equation (2) to the estimated bolometric luminosity curve, we can determine the constant for each SN. Assuming that radioactive materials were powering the latetime photometric evolution, we estimated the ${ }^{56} \mathrm{Ni}$ mass by comparing the luminosity curves of each SN and of SN 1987A with the following equation:

$$
M_{56 \mathrm{Ni}}(\mathrm{SN})=M_{56 \mathrm{Ni}}(87 \mathrm{~A}) \times \frac{\text { Const }_{\mathrm{SN}}}{\text { Const }_{87 \mathrm{~A}}} M_{\odot} .
$$

If we can estimate luminosities at one epoch during the radioactive decay phase, we can estimate the ${ }^{56} \mathrm{Ni}$ mass in principle. The estimated ${ }^{56} \mathrm{Ni}$ mass is listed in the last column of Table 6. Since several bands are lacking for SN 2006bc during the radioactive decay phase, the estimated ${ }^{56} \mathrm{Ni}$ mass is based only on the HST data. Since the quantities for SN $2002 \mathrm{hh}$ are derived from the bolometric luminosity and flux densities of the SN and the light echo, the estimated values might have $>30 \%$ uncertainties. As will be discussed later, the ${ }^{56} \mathrm{Ni}$ mass of SN $2002 \mathrm{hh}$ might go down by $\sim 80 \%$ (i.e., $\sim 2.8 M_{\odot}$ ) when we consider the contribution from the light echo to the total flux.

\subsection{Light Echoes and Environment Densities}

Light echoes are created when the flash from the SNe scatters off dust in their environments, which can be interstellar or

Table 5

The Magnitude Decline Rate ( $\gamma_{\text {band }}$ ) of Type II SNe

\begin{tabular}{|c|c|c|c|c|c|c|c|c|c|}
\hline$\gamma_{\text {band }}$ & Epoch & SN1999bw & SN2002hh & SN2003gd & SN2004et & SN2005cs & SN2006bc & SN1987A & SN1999em \\
\hline \multirow[t]{4}{*}{$\gamma_{V}$} & $30-100$ & $\ldots$ & $\ldots$ & $\ldots$ & 0.764 & 0.067 & $\ldots$ & $\ldots$ & 0.400 \\
\hline & $150-300$ & $\ldots$ & 0.972 & 0.780 & 1.006 & 0.277 & 0.709 & 0.980 & 0.932 \\
\hline & $300-800$ & $\ldots$ & $\ldots$ & $\ldots$ & 1.268 & 1.069 & 0.333 & 1.469 & 0.985 \\
\hline & $>800$ & 0.064 & 0.008 & $\ldots$ & 0.173 & 0.506 & $\ldots$ & 0.562 & $\ldots$ \\
\hline \multirow[t]{4}{*}{$\gamma_{R}$} & $30-100$ & $\ldots$ & 0.147 & $\ldots$ & 0.417 & -0.591 & $\ldots$ & $\ldots$ & -0.030 \\
\hline & $150-300$ & $\ldots$ & 0.971 & 1.211 & 1.014 & 0.684 & $\ldots$ & 0.855 & 0.945 \\
\hline & $300-800$ & $\ldots$ & $\ldots$ & 1.453 & 1.509 & 1.135 & $\ldots$ & 1.640 & 1.126 \\
\hline & $>800$ & $\ldots$ & $\ldots$ & 0.052 & $\ldots$ & $\ldots$ & $\ldots$ & 0.528 & $\ldots$ \\
\hline \multirow[t]{4}{*}{$\gamma_{I}$} & $30-100$ & $\ldots$ & $\ldots$ & 0.798 & 0.105 & -0.856 & $\ldots$ & $\ldots$ & -0.269 \\
\hline & $150-300$ & $\ldots$ & 0.968 & 1.295 & 0.918 & 0.771 & 0.669 & 0.946 & $\ldots$ \\
\hline & $300-800$ & $\ldots$ & 0.135 & $\ldots$ & 1.454 & 1.002 & 0.468 & 1.661 & 1.126 \\
\hline & $>800$ & $\ldots$ & 0.049 & 0.052 & 0.126 & $\ldots$ & $\ldots$ & 0.565 & $\ldots$ \\
\hline \multirow[t]{4}{*}{$\gamma_{J}$} & $30-100$ & $\ldots$ & $\ldots$ & $\ldots$ & -0.467 & -1.164 & $\ldots$ & $\ldots$ & -0.371 \\
\hline & $150-300$ & $\ldots$ & 1.256 & $\ldots$ & 1.497 & 1.071 & $\ldots$ & 1.192 & 1.158 \\
\hline & $300-800$ & $\ldots$ & $\ldots$ & $\ldots$ & 1.016 & 1.017 & 0.493 & 1.528 & $\ldots$ \\
\hline & $>800$ & $\ldots$ & 0.007 & $\ldots$ & 0.117 & $\ldots$ & $\ldots$ & 0.809 & $\ldots$ \\
\hline \multirow[t]{4}{*}{$\gamma_{H}$} & $30-100$ & $\ldots$ & $\ldots$ & $\ldots$ & -0.269 & -0.911 & $\ldots$ & $\ldots$ & -0.428 \\
\hline & $150-300$ & $\ldots$ & 1.316 & $\ldots$ & 1.438 & $\ldots$ & $\ldots$ & 1.305 & 1.444 \\
\hline & $300-800$ & $\ldots$ & $\ldots$ & $\ldots$ & 1.098 & $\ldots$ & 0.555 & 1.386 & $\ldots$ \\
\hline & $>800$ & $\ldots$ & 0.014 & $\ldots$ & 0.003 & $\ldots$ & $\ldots$ & 0.857 & $\ldots$ \\
\hline \multirow[t]{4}{*}{$\gamma_{K}$} & $30-100$ & $\ldots$ & $\ldots$ & $\ldots$ & -0.245 & -0.784 & $\ldots$ & $\ldots$ & -0.425 \\
\hline & $150-300$ & $\ldots$ & 1.286 & $\ldots$ & $\ldots$ & 1.790 & $\ldots$ & 1.578 & 1.482 \\
\hline & $300-800$ & $\ldots$ & $\ldots$ & $\ldots$ & 1.177 & $\ldots$ & 0.493 & 1.594 & $\ldots$ \\
\hline & $>800$ & $\ldots$ & 0.011 & $\ldots$ & -0.510 & $\ldots$ & $\ldots$ & 0.624 & $\ldots$ \\
\hline
\end{tabular}

Note. The $\gamma_{\text {band }}$ is the dropping magnitude per 100 days. The values in boldface are derived from the data taken by our observations and archival HST data. 
Table 6

Luminosity, Temperature, Emitting Radius, and ${ }^{56} \mathrm{Ni}$ Mass

\begin{tabular}{lccccc}
\hline \hline SN Name & $\begin{array}{c}\text { Epoch } \\
(\text { day })\end{array}$ & $\begin{array}{c}L_{\text {hot }} \\
\left(\times 10^{6} L_{\odot}\right)\end{array}$ & $\begin{array}{c}T_{\text {eff }} \\
(\mathrm{K})\end{array}$ & $\begin{array}{c}R_{\text {hot }} \\
\left(\times 10^{13} \mathrm{~cm}\right)\end{array}$ & $\begin{array}{c}M\left({ }^{56} \mathrm{Ni}\right) \\
\left(\times 10^{-2} M_{\odot}\right)\end{array}$ \\
\hline SN2002hh & 150 & 238 & 5490 & 119 & 14 \\
& 300 & 39.9 & 6170 & 38.6 & $\ldots$ \\
SN2003gd & 300 & 2.4 & 5930 & 10 & 0.8 \\
SN2004et & 150 & 92.9 & 7040 & 45.1 & 5.9 \\
& 300 & 12.6 & 7200 & 15.9 & $\ldots$ \\
SN2005cs & 300 & 1.50 & 7250 & 5.41 & $\ldots$ \\
SN2006bc & 600 & 0.3 & 6800 & 2.84 & 2.7 \\
SN1987A & 150 & 86.5 & 6030 & 59.4 & 7.3 \\
& 300 & 25.8 & 7130 & 23.2 & $\ldots$ \\
SN1999em & 150 & 22.0 & 5560 & 35.2 & $\ldots$ \\
& 300 & 6.1 & 5220 & 21.1 & $\ldots$ \\
\hline
\end{tabular}

Note. The uncertainties of $L_{\text {hot }}$ and $T_{\text {eff }}$ are $\sim 20 \%$ and $\sim 500 \mathrm{~K}$.

circumstellar. The timing of the light echo depends on the location of the dust with respect to the SN. However, light echoes at late times in the SN light curve are easier to detect because the increased light due to the $\mathrm{SN}$ is more apparent when the SN light has decayed significantly.

The intensity of the light echo depends on the density of the surrounding medium. Using the scattering optical depth of the light echo given by Romaniello et al. (2005),

$$
\begin{aligned}
\tau_{\text {echo }, \text { sca }}(\lambda)= & 7.6 \times 10^{-5} \frac{5.8 \times 10^{21}}{k} \frac{R(\lambda)}{3.1} \frac{\delta t_{\mathrm{SN}}(\lambda)}{100 \text { days }} \\
& \times \frac{\omega(\lambda)}{0.6} \frac{n(\mathrm{H})}{1 \mathrm{~cm}^{-3}} \frac{n(\mathrm{O})}{n(\mathrm{O})_{\odot}},
\end{aligned}
$$

where $k=N_{\mathrm{H}} / E(B-V)$ is the ratio of total neutral hydrogen column density to color excess at solar metallicity $(k=5.8 \times$ $10^{21} \mathrm{~cm}^{-2} \mathrm{mag}^{-1}$; Bohlin et al. 1978), $\delta t_{\mathrm{SN}}$ is the duration of the burst of the SN ( $\sim 100$ days in the case of Type II-P), and $\omega(\lambda)$ is the grain albedo ( $\simeq 0.6$ at optical wavelength; e.g., Mathis et al. 1977). Using Equation (2), $\tau_{\text {echo,sca }}(V)$ is estimated to be $1.205 \times 10^{-4} n_{\mathrm{H}}$, adopting $\omega(V)=0.6$ and $\delta t_{\mathrm{SN}}(\lambda)=100$ days. Sparks (1994) argued that the difference between peak $\left(M_{\mathrm{SN}}\right)$ and light-echo magnitudes $\left(M_{\text {echo }}\right)$ is

$$
M_{\mathrm{echo}} \simeq M_{\mathrm{SN}}+0.5-2.5 \log \left(\tau_{\mathrm{echo}, \mathrm{sca}}\right) .
$$

If a light echo is associated with an $\mathrm{SN}, M_{\text {echo }}(V)-M_{\mathrm{SN}}(V) \simeq$ 10.3-2.5log $n(\mathrm{H})$. Using the light curves, we can measure the magnitude difference in SNe with light echoes and estimate the density $n(\mathrm{H})$.

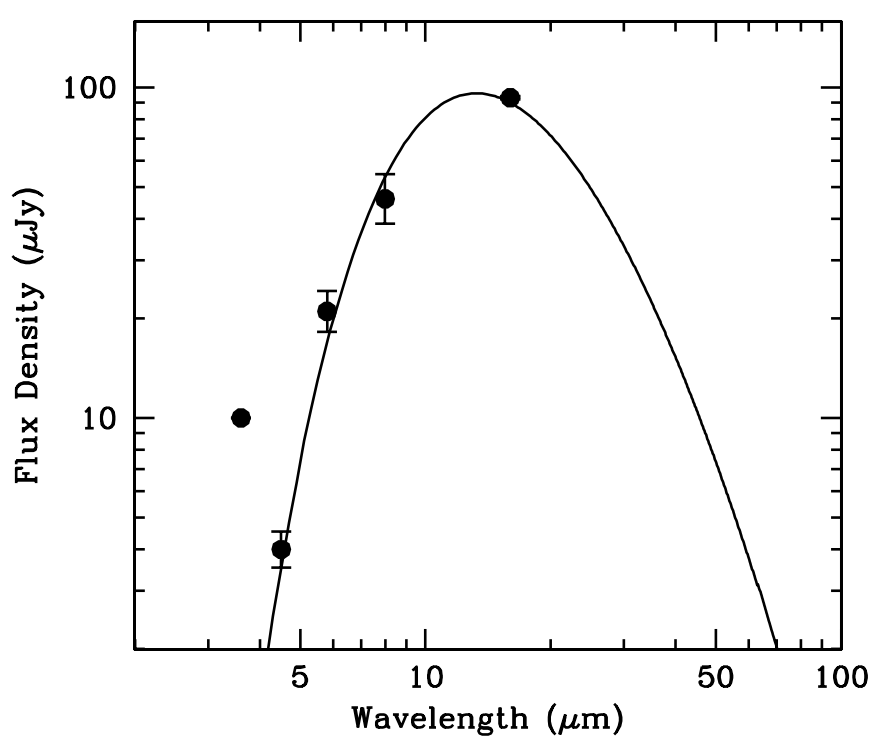

Figure 4. Mid-IR SED of SN 1999bw at days $3316(3.6 / 4.5 / 5.8 / 8.0 \mu \mathrm{m})$ and $3331(16 \mu \mathrm{m})$. The filled circles are observations. The thick line is the result of modified blackbody fitting.

\section{RESULTS FOR INDIVIDUAL SNe}

\subsection{SN 1999bw}

SN 1999bw is a Type II-n SN in NGC 3198 ( $d=13.7$ Mpc; Freedman et al. 2001) and was discovered on JD.2451288.7. The position measured from our HST observation is in agreement with Sugerman et al. (2004) within 0'.5. We detected the light of the $\mathrm{SN}$ in $\mathrm{F} 606 \mathrm{~W}$ and $\mathrm{F} 814 \mathrm{~W}$. The optical spectra at early epochs are dominated by emission lines having full width at half maximum (FWHM) of $\sim 1000-2000 \mathrm{~km} \mathrm{~s}^{-1}$ (Garnavich et al. 1999; Filippenko et al. 1999). With Spitzer/IRAC flux densities and the blackbody fitting method, Sugerman et al. (2004) estimated the emitting radius of $1.6 \times 10^{16} \mathrm{~cm}$ and the expansion velocity of $1000 \mathrm{~km} \mathrm{~s}^{-1}$ in the fifth year after the initial explosion. Dust production has not been measured for this SN so far, most likely because insufficient data exist for such an estimate. Fabbri (2011) measured flux densities in four Spitzer/IRAC bands (days 3316), IRS-PU $16 \mu \mathrm{m}$ (3331), and MIPS $24 \mu \mathrm{m}$ (3320), as listed in Table 7. Based on her results, we estimated a dust mass of $9.2 \times 10^{-5} M_{\odot}$ at a temperature of $\sim 300 \mathrm{~K}$ at day $\sim 3320$ using modified blackbody fitting, assuming amorphous carbon grains with only $0.1 \mu \mathrm{m}$ radius. In the fitting, we adopted the optical constants of Zubko et al. (1996). The best-fit SED is presented in Figure 4.

Smith et al. (2011) reported BVRI magnitudes at the very early plateau phase. They suggested that this object might

\begin{tabular}{|c|c|c|c|c|c|c|c|}
\hline \multirow[t]{2}{*}{ Obs. Date } & \multirow{2}{*}{$\begin{array}{l}\text { Epoch } \\
\text { (day) }\end{array}$} & \multicolumn{6}{|c|}{ Flux Density $(\mu \mathrm{Jy})$} \\
\hline & & $3.6 \mu \mathrm{m}$ & $4.5 \mu \mathrm{m}$ & $5.8 \mu \mathrm{m}$ & $8.0 \mu \mathrm{m}$ & $16 \mu \mathrm{m}$ & $24 \mu \mathrm{m}$ \\
\hline 2008 May 13 & 3316 & $\leqslant 10$ & $4.0 \pm 0.5$ & $21 \pm 3$ & $46 \pm 8$ & . & $\ldots$ \\
\hline 2008 May 17 & 3320 & $\ldots$ & $\ldots$ & $\ldots$ & $\ldots$ & $\ldots$ & $\leqslant 650$ \\
\hline 2008 May 28 & 3331 & $\ldots$ & $\ldots$ & $\ldots$ & $\ldots$ & $93 \pm 16$ & $\ldots$ \\
\hline
\end{tabular}

Table 7

SN 1999bw Spitzer Observations

Notes. The upper limits for the MIPS $24 \mu \mathrm{m}$ data were estimated by measuring the flux density of the faint point source seen $\sim 6^{\prime \prime}$ to the east of the SN position. This was found to have an average brightness of $657 \mu \mathrm{Jy}$ with a standard deviation of $2.5 \%$ over the four epochs, and this approximate value was used as a robust upper limit for the SN brightness at each epoch. This table is taken from Fabbri (2011). 


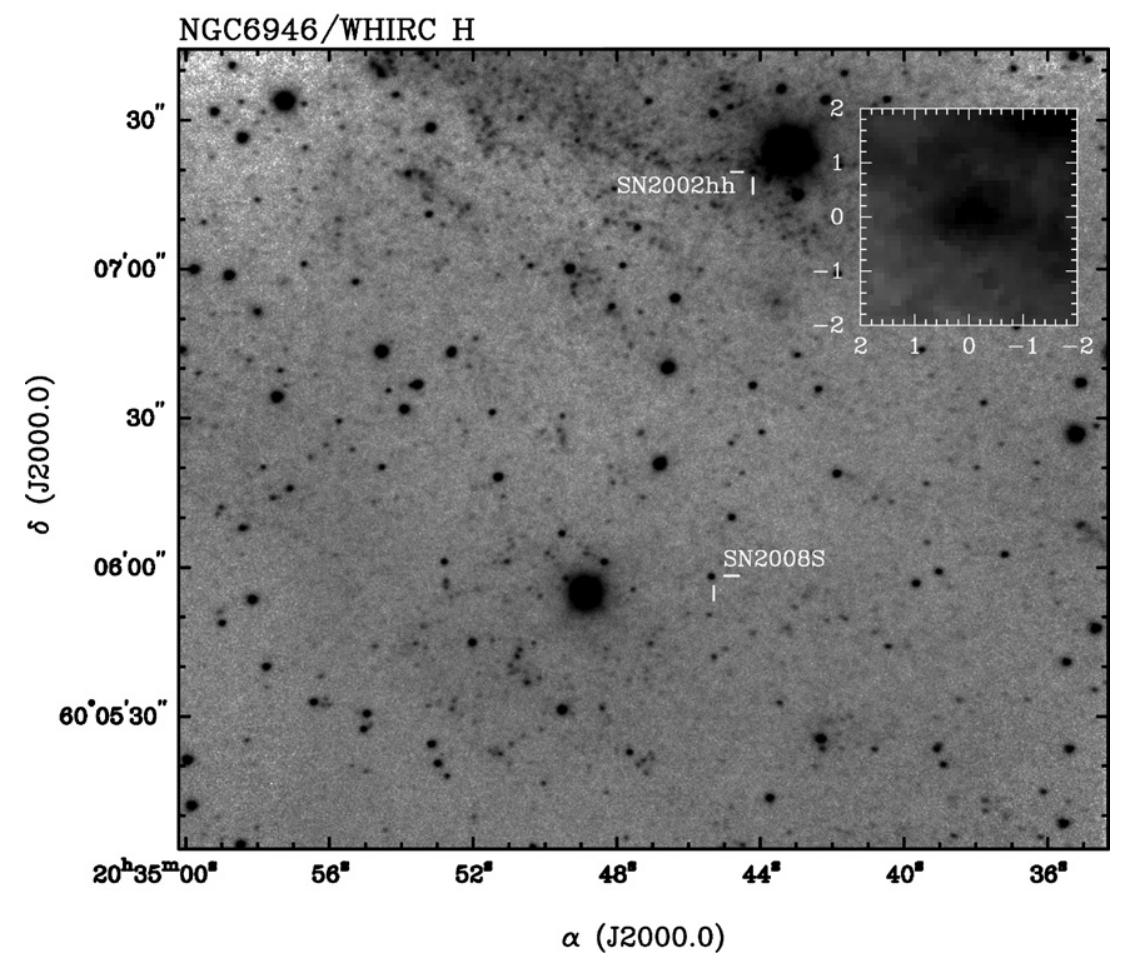

Figure 5. WHIRC view of the SNe 2002hh and 2008S field in the $H$ band. The positions of SNe $2002 \mathrm{hh}$ and $2008 \mathrm{~S}$ are indicated by the white tick marks. A close-up image of SN $2002 \mathrm{hh}$ is presented in the inner box $\left(2^{\prime \prime} \times 2^{\prime \prime}\right)$. The image was taken in a 2008 observing run (PI: M. Meixner).

be an impostor from statistical study of expansion velocities of Type II SNe and luminous blue variables (LBVs) based on optical spectra. They estimated a peak absolute $R$-band magnitude of -12.65 using optical spectra at day 4 , which lies between those of P Cygni and $\eta$ Carinae. There is no information on the progenitor star. There is no information on the brightness of the SN during days 150-626, so we estimate the $V$-band magnitude decline rate for $>800$ days. Since the magnitude difference between the peak and day $>1000$ is $\sim 6.6$, the hydrogen density $n(\mathrm{H})$ assuming solar $\mathrm{O}$ abundance (8.69; Lodders 2003) is estimated to be $\sim 30 \mathrm{~cm}^{-3}$, which is larger than typical interstellar gas densities $\left(1-10 \mathrm{~cm}^{-3}\right)$. We might therefore be looking mostly at pre-existing circumstellar material rather than the ISM.

\subsection{SN 2002hh}

SN 2002hh is a Type II-P SN in a spiral arm of the starburst galaxy NGC 6946 ( $d=5.9 \mathrm{Mpc}$; Karachentsev et al. 2000) and was discovered on JD.2452574.5. We detected the light from the SN in HST, NIRI, and WHIRC observations. The SN birth rate in this galaxy is very high, $\sim 0.1 \mathrm{SN} \mathrm{yr}^{-1}$ during the past 100 yr. SNe 1969P and 2008S were observed (Figure 5) within a $2^{\prime}$ radius from $\mathrm{SN} 2002 \mathrm{hh}$. The high-SN formation rate could be a natural consequence of the high star forming activity in NGC 6946.

SN 2002hh is still bright and close to peak brightness even after $\sim 7$ years (Figure 3 ). While at the early plateau phase around day 150 the fading rate roughly followed the ${ }^{56} \mathrm{Co}$ decay power $(0.97$ at $V$ and $I$ and $\sim 1.3$ at $J H K$ ), the fading rates are $\lesssim 0.1$ from day 300 or later. Pozzo et al. (2006) argued that the near-IR excess in this SN is due to an IR light echo from a preexisting, dusty circumstellar medium. In Figure 6, we show the temporal evolution of the SN at F606W ( $V$ band). The 2005 and 2006 images (upper left two panels) were taken by ACS/HRC. In the three middle panels, we show the subtraction images between two different epochs. Two arc structures are seen and appear to be expanding with time. We interpret these structures as light echoes.

Our formula for the density of the environment depends on the metallicity, here measured as the oxygen $(\mathrm{O})$ abundance. Belley \& Roy (1992) investigated the oxygen (O) abundances in $160 \mathrm{H}$ II regions in NGC 6946 and estimated the $\mathrm{O}$ abundance gradient of $\Delta \log (n(\mathrm{O}) / n(\mathrm{H})) / \Delta R=-0.089 \mathrm{dex} \mathrm{kpc}^{-1}$, where $n(\mathrm{O})$ and $n(\mathrm{H})$ are the number densities of oxygen and hydrogen and the extrapolated $\log n(\mathrm{O}) / n(\mathrm{H})+12=9.36$ and $R$ is the distance from the core of the galaxy. SNe 2002hh and 2004et (see below) are located $\sim 5.7$ and $\sim 8 \mathrm{kpc}$ from the center, respectively. When we adopt the solar $\mathrm{O}$ abundance, the $\mathrm{O}$ abundances of SNe 2002hh and 2004et are expected to be 8.85 and 8.65 , respectively.

Since the difference $V$ magnitude between the peak and day 1000 or later is $\sim 3.7, n(\mathrm{H}) \sim 440 \mathrm{~cm}^{-3}$ is required if the light echo is associated with the $\mathrm{SN}$. This hydrogen density is close to relatively dense gas density in such $\mathrm{H}$ II regions rather than that in interstellar space. Welch et al. (2007) suggest that most of the Spitzer mid-IR flux may have come from dust in the star formation region associated with SN 2002hh precursor. We should note that we measured the magnitude of the $\mathrm{SN}$ and the light echo. In the case of SN 2004et, which has a light echo and is a member of NGC 6946, the apparent radius of the SN is $\sim 0^{\prime}$. 1 . If we adopted $00^{\prime} 1$ radius in aperture photometry for $\mathrm{SN}$ $2002 \mathrm{hh}$ and we define that the inner radius of the light echo is $>0$.' $1, \sim 80 \%$ of the total flux is from the light echo as of day 1717 (our HST observations).

Barlow et al. (2005) estimated $0.1-0.15 M_{\odot}$ dust around SN 2002hh after 600 days with radiative transfer modelings based on Spitzer/IRAC/MIPS. However, the minimum emitting radius $\sim 10^{17} \mathrm{~cm}$ is too large for the emitting dust to be forming dust in the SN ejecta. Pozzo et al. (2006) suggest that dust within $\sim 10^{17} \mathrm{~cm}$ would be evaporated. Barlow et al. (2005) therefore 

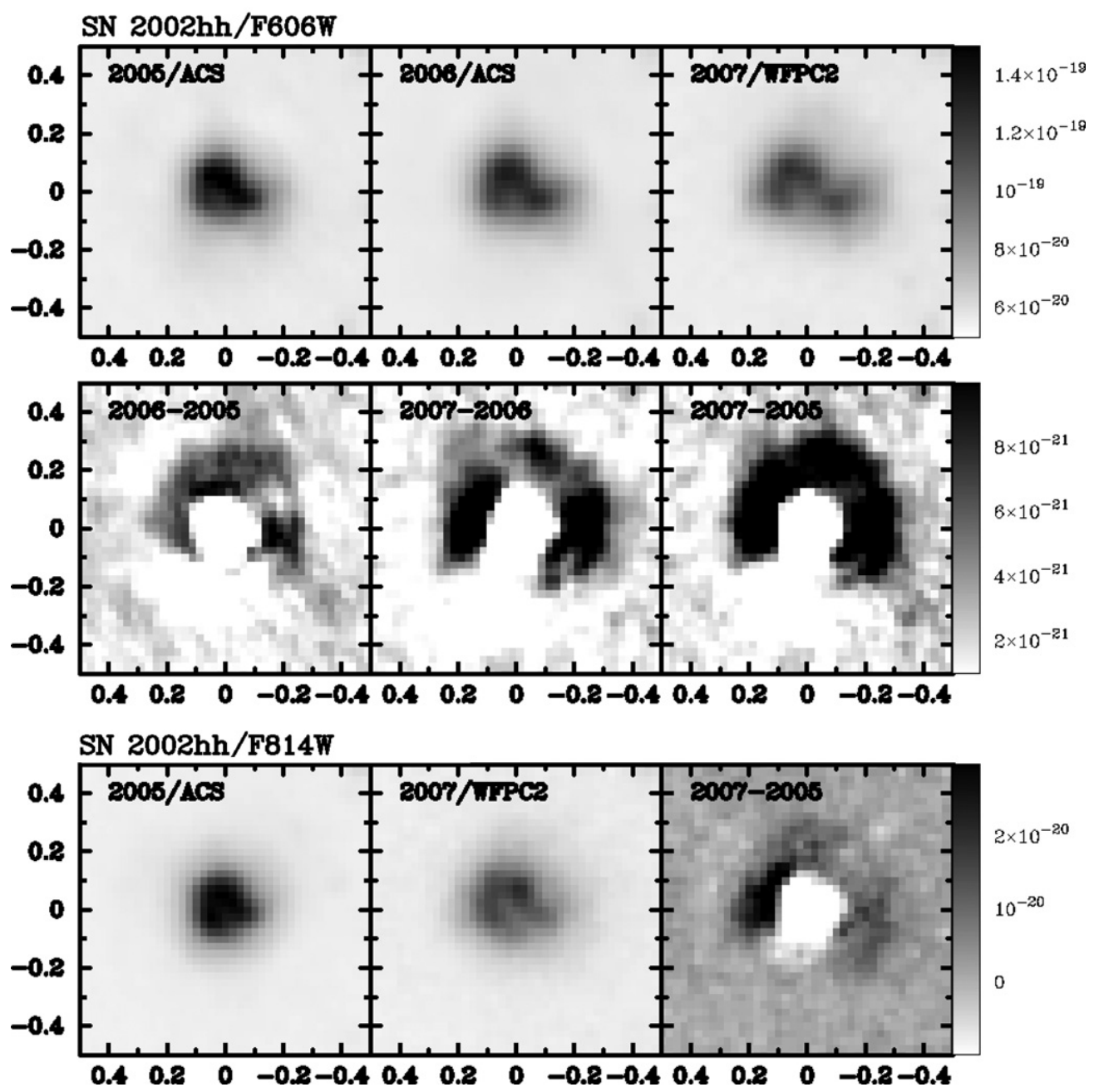

Figure 6. (Top six panels) The temporal evolution of SN 2002hh at F606W band. (Bottom three panels) The temporal evolution of SN 2002hh at F814W band. The F606W difference images are created after PSF matching each band image.

concluded that the emitting dust must have been pre-existing and its origin may be from an enhanced mass loss by the $>10 M_{\odot}$ progenitor.

\subsection{SN $2003 g d$}

SN 2003gd is a Type II-P SN in NGC $628(d=7.2 \mathrm{Mpc}$; Van Dyk et al. 2003) and was discovered on JD.2452803.2. Van Dyk et al. (2003) and Smartt et al. (2004) identified the progenitor with an F606W HST image; the progenitor is a $6-12 M_{\odot}$ red supergiant. Maund \& Smartt (2009) estimated the $V$ - and $I$-band magnitudes of the progenitor using pre-explosion images from the HST and Gemini telescope archives. They estimated $V=$ 25.8 and $I=23.3$ and an initial mass of $6-12 M_{\odot}$.

The $V R$-band fading rates from days 150 to 300 are comparable with the radioactive decay rate as earlier mentioned. But, in days 300-800, the fading rates are much larger than those supported by the ${ }^{56} \mathrm{Co}$ decay power, indicating that dust formation had started from day $\sim 300$ or later. Sugerman et al. (2006) estimated the dust mass of $1.7 \times 10^{-3} M_{\odot}$ at day 499 (clumped dust distribution model).

When we focus on day $\sim 500$ or later, the magnitude is almost constant. This is due to a light echo. Sugerman (2005; 0'.225 inner and 0.'375 outer radii at day 632) and Van Dyk et al. (2006; 0.31 at the same epoch) found the light echo in the F625W band. We detected an arc shaped light echo with $\sim 0$ '.5 radius $\left(5.39 \times 10^{19} \mathrm{~cm}\right.$ at $\left.7.2 \mathrm{Mpc}\right)$ in the same band as shown in Figure 7 . We should note that the shape of the light echo is changing with time. Belley \& Roy (1992) measured the $\mathrm{O}$ abundances in $130 \mathrm{HII}$ regions in NGC 628 and estimated the $\mathrm{O}$ abundance gradient of $\Delta \log (n(\mathrm{O}) / n(\mathrm{H})) / \Delta R=$ $-0.081 \mathrm{dex} \mathrm{kpc}^{-1}$, where the extrapolated $\log n(\mathrm{O}) / n(\mathrm{H})+12=$ 9.2 in the galaxy core. When we adopt an $R$ of $\sim 4.5 \mathrm{kpc}$ and $R(R)=2.32, M_{\text {echo }}(R)-M_{\mathrm{SN}}(R) \simeq 10.6-2.5 \log n(\mathrm{H})$. Since the difference magnitude between the peak and day 1998 is $\sim 10.97$, $n(\mathrm{H})$ is $\sim 1.4 \mathrm{~cm}^{-3}$, which corresponds to a typical ISM density.

\subsection{SN 2004et}

SN 2004et is a Type II-P SN in NGC 6946 discovered on JD.2453217.5. We successfully detected the SN at all the observed bands.

The light curves and fading rates at all bands are very similar to those of SNe 1987A and 1999em except for day $\sim 1000$ or later. Up to 1000 days after the initial explosion, the brightness at both optical and infrared wavelength bands was monotonically fading, but from $\sim 1200$ days it starts to increase again, in particular in the $K$ band. Our HST observations were taken just before and after the beginning of this brightening. Kotak et al. (2009) argued that this phenomenon is caused by an interaction between the SN ejecta and the surrounding ISM. On the other hand, B. E. K. Sugerman et al. (2012, in preparation) propose 


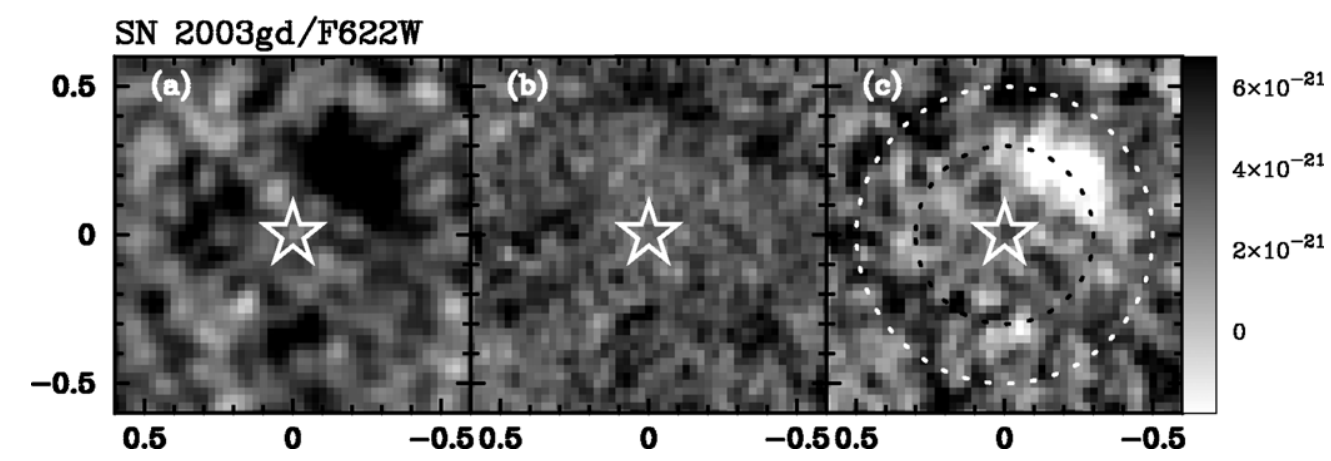

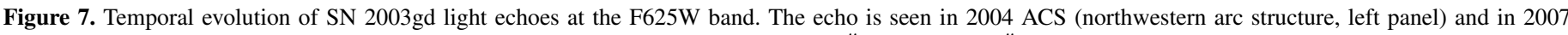

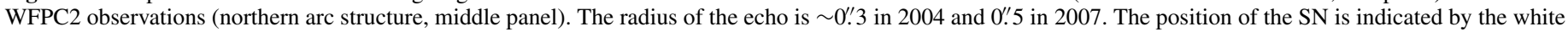
stars. The difference images in right panel are created after PSF matching each band image. The radii of broken black and white are 0. .' 3 and $0 .{ }^{\prime} 5$, respectively.

a light echo as an explanation for the re-brightening. Since the difference of $M(V)$ between the plateau and day $\sim 1000$ is $\sim 10.53, n(\mathrm{H})$ is $\sim 1.2 \mathrm{~cm}^{-3}$. The magnitude after day $\sim 1000$ is consistent with the light-echo interpretation.

With the bolometric light curve Sahu et al. (2006) estimated a ${ }^{56} \mathrm{Ni}$ mass of $\sim 0.06 M_{\odot}$. The progenitor mass is $15-24.5 M_{\odot}$ (Li et al. 2005; Utrobin \& Chugai 2009). Fabbri et al. (2011) estimated an SN produced dust mass of $4 \times 10^{-4}$ to $4 \times 10^{-3} M_{\odot}$ using SED fits of combined Spitzer and optical/near-IR data. There are active star forming regions around SN 2004et. Like SNe 2002hh and 2008S (but an SN impostor, see below), the progenitor of SN 2004et would have formed in a metal-rich environment.

\subsection{SN $2005 c s$}

SN 2005cs is a Type II-P SN in NGC $5194(d=7.1 \mathrm{Mpc}$; Takáts \& Vinkó 2006) and was discovered on JD.2453550.4. We note that the position measured from the F606W image differs significantly from the position we used for observing, i.e., the SIMBAD position. As a consequence, SN 2005cs was unfortunately on the edge of the field for the observations we report and was entirely missed by our NIC2 measurement in 2008. SN 2005cs is $\sim 0{ }^{\prime} 1$ northeast from the bright cluster (Li et al. 2006). Due to its faintness, we cannot detect the SN in the NIC2/F160W and 205W bands.

The VRI fading rate at day 150-300 is much smaller than the ${ }^{56}$ Co decay power. Pastorello et al. (2009) argued that such flattening could be formed by a radiation flow generated in the warmer inner ejecta, which propagates throughout the transparent cooler external layers, and contributes to the light curve as an additional source. The light curves and magnitude decline rates beyond day $\sim 300$ are comparable to SNe 2004et and 1987A, suggesting that dust formation may have occurred. Fabbri (2011) gave an upper limit to the dust mass $=3.1 \times 10^{-3} M_{\odot}($ O-rich case $)$ from the detection limit of a Gemini/Michelle $10 \mu \mathrm{m}$ image obtained in 2007 (PI: M. J. Barlow).

Li et al. (2006) detected the progenitor from the HST ACS images, and they estimated $V>25.5$ and $I>24.14$. Maund et al. (2005) and Li et al. (2006) reported the detection of the progenitor using the archival HST ACS images. They argued that the progenitor is a $\sim 7-12 M_{\odot}$ red super giant. Pastorello et al. (2009) obtained similar results $\left(8-15 M_{\odot}\right)$. Pastorello et al. (2009) also estimated that the explosion energy is $\sim 3 \times 10^{50} \mathrm{erg}$ (cf. $\sim 10^{51} \mathrm{erg}$ in typical Type II SNe) and a ${ }^{56} \mathrm{Ni}$ mass of (2.8-4.2) $\times 10^{-3} M_{\odot}$. They measured a low expansion velocity $\left(1000-2000 \mathrm{~km} \mathrm{~s}^{-1}\right)$ in optical and near-IR spectra. The flattening of the light curve in the last two epoch measurements suggests a light echo. Since the difference between the peak and day 1006 is 11.2 in the $V$ band, the ISM density is $\sim 0.4 \mathrm{~cm}^{-3}$ assuming that the $\mathrm{SN}$ has a light echo and the SN light after day 1006 is partially from the light echo.

\section{6. $S N 2006 b c$}

SN 2006bc is either a Type II-L (Gallagher et al. 2010) or Type II-P (Brown et al. 2009) SN in NGC 2397 ( $d=16$ Mpc; Mihos \& Bothun 1997) and was discovered on JD.2453819.4. The Swift telescope detected the SN light at an early epoch and the $V$ magnitude was $\sim 17.5$ at day 15.8 (Brown et al. 2009). We detected the SN light in all bands. Smartt et al. (2009) give an upper limit for the progenitor mass of $12 M_{\odot}$ for SN 2006bc

SN 2006bc is surrounded by an H II region; the optical spectra taken by Gemini-N/GMOS show the complex of the SN and $\mathrm{H}$ II region components (Gallagher et al. 2011). Gallagher et al. (2011) extracted the SN component by multi-Gaussian fitting, and they confirmed that the $\mathrm{H} \alpha$ line profile is blueward shifting as the SN becomes older suggesting dust production. An IR-excess has been measured in the Spitzer IRAC bands and SED fitting by Gallagher et al. (2011) indicates a dust mass of $9 \times 10^{-4} M_{\odot}$. The fading rates are much lower than the typical ${ }^{56} \mathrm{Co}$ decay power and our estimated ${ }^{56} \mathrm{Ni}$ mass of $2.7 \times 10^{-2} M_{\odot}$ is lower than average in our sample. A flattening of the light curve between days 400 and 500 suggests a short duration light echo. The estimated density of the matter in this light echo is $\sim 36 \mathrm{~cm}^{-3}$ from the difference $V$ magnitude $=6.4$ between day 15.8 and 694 .

\subsection{SN Impostor: SN $2008 S$}

SN 2008S is regarded as an SN impostor in NGC 6946 and was discovered around JD.2454498 (Arbour \& Boles 2008). $E(B-V)$ by the Galaxy is estimated to be 0.34 from reddening maps of Schlegel et al. (1998). Botticella et al. (2009) estimated the extinction by the host galaxy $A_{V} \sim 1$ ( 1.13 by the Galaxy) by comparing their light-echo model with the observed SED at 17 days. In this paper, we corrected only the extinction by the Galaxy with $E(B-V)=0.34$. Our $J H K$ light curves show that SN 2008S is quite different from Type II SNe: for example, there is no plateau phase and no clear level-off around day $\sim 100$. The nature of the progenitor star is under debate. Botticella et al. (2009) argue that SN 2008S may have evolved from a 6-8 $M_{\odot}$ asymptotic giant branch (AGB) star and formed not by Fe-core collapse but by $\mathrm{O}-\mathrm{Ne}-\mathrm{Mg}$ core collapse by electron capture during the AGB phase, while Smith et al. (2011) argue for a 
Table 8

Derived Quantities for our Targets in this Study and our SEEDS Sample

\begin{tabular}{|c|c|c|c|c|c|c|c|c|c|}
\hline $\begin{array}{l}\mathrm{SN} \\
\text { Name }\end{array}$ & Type & $\begin{array}{l}\text { Distance } \\
(\mathrm{Mpc})\end{array}$ & $\begin{array}{c}E(B-V) \\
(\mathrm{MW}+\text { Host })\end{array}$ & $\begin{array}{l}M_{\text {prog }} \\
\left(M_{\odot}\right)\end{array}$ & $\begin{array}{c}M^{\left({ }^{56} \mathrm{Ni}\right)} \\
\left(\times 10^{-2} M_{\odot}\right)\end{array}$ & $\begin{array}{l}M_{\text {dust }^{\mathrm{a}}} \\
\left(M_{\odot}\right)\end{array}$ & $\begin{array}{l}\text { ISM Dens. } \\
\left(\mathrm{cm}^{-3}\right)\end{array}$ & $\begin{array}{l}\text { Light } \\
\text { Echo? }\end{array}$ & $\begin{array}{c}\text { References for } \\
M_{\text {prog }} / M\left({ }^{56} \mathrm{Ni}\right) / M_{\text {dust }}\end{array}$ \\
\hline 1999bw & $\begin{array}{l}\text { II-n or } \\
\text { impostor }\end{array}$ & 13.7 & 0.01 & $?$ & $?$ & $\sim 10^{-4}$ & $\sim 30$ & No & (1) \\
\hline 2002hh & II-P & 5.9 & 1.97 & $>10$ & $<14$ & $0.1-0.15^{b}$ & $\sim 400$ & Yes & $(1),(2)$ \\
\hline $2003 \mathrm{gd}$ & II-P & 7.2 & 0.18 & $6-12$ & 0.8 & $4 \times 10^{-5}-1.7 \times 10^{-3}$ & $\sim 1.4$ & Yes & $(1),(3),(4)$ \\
\hline 2004et & II-P & 5.9 & 0.41 & $15-24.5$ & 5.9 & $1.5 \times 10^{-4}-1.5 \times 10^{-3}$ & $\sim 1.2$ & Yes & $(1),(5),(6),(7)$ \\
\hline $2005 \mathrm{cs}$ & II-P & 7.1 & 0.14 & $7-12$ & 0.5 & $\leqslant 3.1 \times 10^{-3}$ & $\sim 0.4$ & No & $(1),(8),(9),(10)$ \\
\hline $2006 b c$ & II-P or II-L & 16 & 0.52 & $\lesssim 12$ & 2.7 & $9 \times 10^{-4}$ & $\sim 36$ & Yes & $(1),(11),(12)$ \\
\hline 2007it & II-P & 11.7 & 0.13 & $\widetilde{16}-27$ & 9 & $\sim 10^{-4}$ & $\sim 19$ & Yes & (13) \\
\hline 2007od & II-P & 24.5 & 0.13 & $?$ & 0.26 & $4 \times 10^{-4}$ & $\sim 3.6$ & Yes & (14) \\
\hline 1987A & II-P & 50 & 0.19 & $16-22$ & 7.5 & $(3-5) \times 10^{-4}-1.3 \times 10^{-3}$ & $\sim 1.5$ & Yes & (15) \\
\hline $1999 \mathrm{em}$ & II-P & 7.83 & 0.10 & $12-14$ & 1.9 & $\gtrsim 1 \times 10^{-4}$ & $\ldots$ & No & $(16),(17)$ \\
\hline $2008 S$ & impostor & 5.9 & $0.34^{\mathrm{c}}$ & $6-8$ & 0.14 & $<0.02^{\mathrm{b}}$ & $?$ & Yes & $(18),(19)$ \\
\hline
\end{tabular}

Notes.

a The estimated dust mass is based on mid-IR data.

${ }^{b}$ Most of the dust is pre-existing material.

c The extinction by the host galaxy is not included.

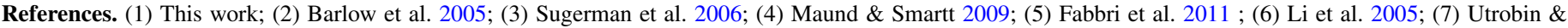

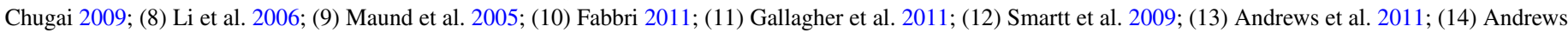
et al. 2010; (15) Ercolano et al. 2007; (16) Elmhamdi et al. 2003; (17) Kozasa et al. 2009; (18) Wesson et al. 2010; (19) Botticella et al. 2009.

high mass progenitor $\left(>20 M_{\odot}\right)$ for this class of object such as SN2008S. The ${ }^{56} \mathrm{Ni}$ mass of SN $2008 \mathrm{~S}$ has the smallest Ni mass estimated for a Type II SNe; the value is $1.4 \times 10^{-3} M_{\odot}$ (Botticella et al. 2009).

Wesson et al. (2010) estimated the dust mass-loss rate of the progenitor of SN $2008 \mathrm{~S}$ to be $5 \times 10^{-7} M_{\odot} \mathrm{yr}^{-1}$ and the total dust injection into the ISM to be $<0.01 M_{\odot}$. The large infrared excess on day 17 could only be an echo, as there would be no way for dust in the necessary quantity to have formed in such a short time. The SED on day 180 could be well matched by models using the same shell geometry as day 17 , without any need for new dust formation.

\section{DISCUSSION}

In Table 8, we summarize the derived physical quantities of our sample. ${ }^{56} \mathrm{Ni}$ mass (sixth column of this table) and ISM density (eighth column) are from this work. The other quantities are from the literature listed in the last column.

The behaviors of the SN light curves are affected mainly by (1) the explosion energy, (2) the ISM density $\left(n_{\mathrm{H}}\right)$, and (3) the formed dust mass $\left(M_{\text {dust }}\right)$ in the SNe. Because the explosion energy determines the peak luminosity of the $\mathrm{SNe}$, the surrounding ISM density/distribution and the dust affects the light curves at $\gtrsim 1000$ days and at $\gtrsim 300$ days, respectively. Relations between progenitor mass $\left(M_{\text {prog }}\right)$ and ${ }^{56} \mathrm{Ni}$ mass and explosion energy are known for massive $\left(>20 M_{\odot}\right)$ Type II SNe (e.g., Maeda et al. 2010); however, relations among the ${ }^{56} \mathrm{Ni}$ mass, $M_{\text {dust }}, M_{\text {prog }}$, and ISM density are unknown for less massive Type II SNe. Through this work, we have estimated the ISM density and the ${ }^{56} \mathrm{Ni}$ mass for our sample using our HST data. Using the theoretical radiative transfer code MOCASSIN (Ercolano et al. 2005), we have estimated dust masses for our sample except for SNe 1999bw, 1999em, and 2005cs. The dust masses listed in this table are based on mid-IR data and the value is as of $<800$ days. Here, we will examine relations among ${ }^{16} \mathrm{Ni}$ mass, $M_{\text {dust }}, M_{\text {prog }}$, ISM density, and $E(B-V)$.

\section{1. ${ }^{56}$ Ni Mass versus $M_{\text {prog }}$}

In Figure 8(a), we present the relation between the ${ }^{56} \mathrm{Ni}$ and the progenitor masses $M_{\text {prog. }}$. In principle, there is a relationship among the amount of ${ }^{56} \mathrm{Ni}$ yield in stars, the progenitors masses, the initial abundances, and the metallicity. The metallicity of our sample is similar; we assume that the effects of metallicity in nucleosynthesis would be therefore negligible. Maeda et al. (2010) find relationships between the predicted ${ }^{56} \mathrm{Ni}$ mass (of order $10^{-2} M_{\odot}$ ), the SN progenitor masses, and the kinematic energy of the explosion $\left(1 \sim 100 \times 10^{51} \mathrm{erg} \mathrm{s}^{-1}\right)$; however, they focused on Type II SNe evolved from $>20$ to $50 M_{\odot}$ progenitors (hypernova). We expected a similar relationship between the ${ }^{56} \mathrm{Ni}$ and progenitor masses in Type II SNe with progenitor masses of $\sim 10-25 M_{\odot}$. Figure 8 (a) strongly supports the previous works. As mentioned earlier, the estimated ${ }^{56} \mathrm{Ni}$ mass might have large uncertainties. When we exclude the data of SN 2002hh (due to the large uncertainty of the ${ }^{56} \mathrm{Ni}$ mass), $M\left({ }^{56} \mathrm{Ni}\right)$ represents $0.31 \times 10^{-2} \times M_{\text {prog. }}\left(M_{\odot}\right)$. The ${ }^{56} \mathrm{Ni}$ mass derived from the bolometric luminosity could be an effective tool to estimate the $\mathrm{SN}$ progenitor mass.

\section{2. $n_{H}$ versus $E(B-V)$}

Figure 8(b) shows the relation between ISM density $n_{\mathrm{H}}$ and the extinction $E(B-V)$. We added information on SNe 2007it (Andrews et al. 2010) and 2007od (Andrews et al. 2011), which are targets in our SEEDS project. Andrews et al. estimated the nickel mass and dust mass and found the light echo using HST observations. The ISM densities around SNe 2007it and 2007od are derived in the same way as in the other SNe, using the difference $V$-band magnitude between plateau and day 922 in 2007od (7.12) and day 811 in 2007it (8.9). For both objects, we assume solar metallicity.

There is no correlation between them. This can be explained by the different light-echo geometries (shape, size, inclination angle, etc.). For example, modeling of the possible light-echo geometries in SN 2004et currently suggests an hourglass-shaped nebula similar in size to that discovered around SN 1987A, 

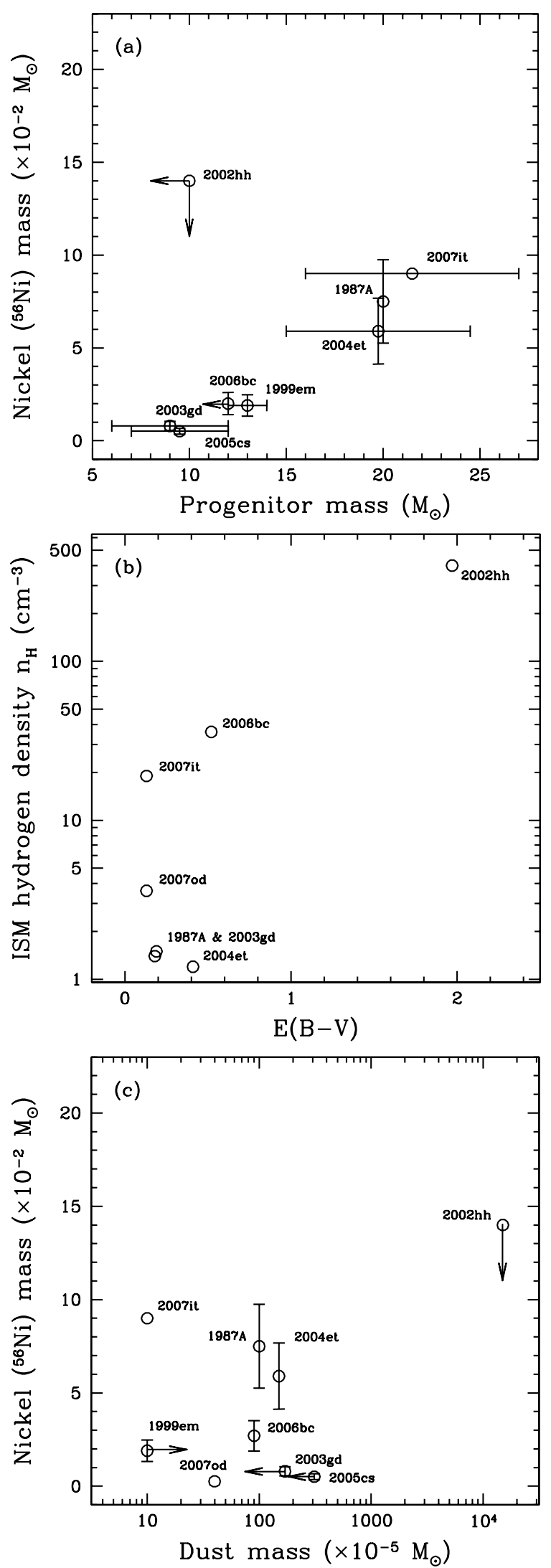

Figure 8. (Upper panel) The relationship between nickel $M\left({ }^{56} \mathrm{Ni}\right)$ and progenitor masses $M_{\text {prog. }}$. (Middle panel). The relationship between ISM hydrogen density and $E(B-V)$. (Lower panel) The relationship between nickel and dust masses.

but oriented such that we are looking down the symmetry axis (B. E. K. Sugerman et al. 2012, in preparation).

\section{3. ${ }^{56} \mathrm{Ni}$ Mass versus $M_{\mathrm{dust}}$}

Figure 8 (c) shows the relation between the ${ }^{56} \mathrm{Ni}$ mass and the dust mass $M_{\text {dust }}$. The dust mass in this diagram is the maximum value in an early phase within 1-3 yr. If the correlation between the ${ }^{56} \mathrm{Ni}$ and progenitor masses is true and we exclude SN 2002hh, where we observed the pre-existing dust rather than SN origin dust, there is no relation or a very weak relation between the dust and progenitor masses within $\sim 30 M_{\odot}$. This diagram also implies that Type II origin dust mass ranges from $\sim 10^{-4}$ up to $10^{-2} M_{\odot}$ per SN at days $<800$ despite the progenitor mass.

The origin of dust in high- $z$ galaxies is a hotly debated issue stemming from both observational and theoretical studies since Bertoldi et al. (2003) discovered large amounts of dust $(\sim 4 \times$ $\left.10^{8} M_{\odot}\right)$ in QSO J1148+5251 $(z=6.4, \sim 900 \mathrm{Myr})$. At the present epoch in our Galaxy, Type II SNe and AGB stars are the main dust producers (Gehrz 1989). In young galaxies, AGB stars are not likely to contribute significantly to dust production. Such low-mass stars proceed too slowly toward the AGB phase to produce dust within $1 \mathrm{Gyr}$. For example, $\sim 1 M_{\odot}$ single stars with solar metallicity take $\sim 10$ Gyr to evolve into thermally pulsing AGB (TP AGB). If the initial mass is $\sim 5 M_{\odot}$, these stars can evolve into TP AGB stars and might be able to be the main dust producers in young galaxies within $\sim 1$ Gyr (Valiante et al. 2009). However, massive stars can evolve into Type II SNe in timespans under 20 Myr. Theoretical ISM dust models by Dwek \& Cherchneff (2011) predict that an average $20 M_{\odot}$ (initial mass) $\mathrm{SN}$ has to make at least $\sim 0.1-1 M_{\odot}$ of dust in order to be a viable source for the dust found in the QSO $\mathrm{J} 1148+5251$. However, the ${ }^{56} \mathrm{Ni}$ mass $-M_{\text {dust }}$ diagram does not follow the prediction of Dwek \& Cherchneff (2011).

\section{SUMMARY}

We performed $B V R I J H K$-band photometry of six Type II $\mathrm{SNe}$ at late epochs (days >500) using HST and ground-based telescopes to investigate their natures and relations between dust mass, ${ }^{56} \mathrm{Ni}$, ISM density, and progenitor mass. Most of our HST observations are successful in detecting the light from the $\mathrm{SNe}$ alone and in measuring magnitudes with less contamination from nearby stars. Combining our data with previously published data, we showed light curves at VRIJHK bands and estimate the declining magnitude rates at each band at four different phases. These light curves and other data indicate that dust is forming in our targets from days $~ 300$ to 400, supporting SN dust formation theory. We estimated the ISM or circumstellar density around SNe 2002hh, 2003gd, 2004et, and 2006bc. The light-echo density around SN 2002hh is higher than a typical ISM density. The ISM density around other SNe is $1-70 \mathrm{~cm}^{-3}$. We estimated ${ }^{56} \mathrm{Ni}$ masses $\left(0.5-14 \times 10^{-2} M_{\odot}\right)$ by comparing their bolometric luminosity with SN 1987A and we find that it correlates with progenitor mass. This relation supports the work by Maeda et al. (2010), who focus on Type II hyper $\mathrm{SNe}\left(>20 M_{\odot}\right)$. The dust mass does not appear to be correlated with ${ }^{56} \mathrm{Ni}$ mass among the seven $\mathrm{SNe}$.

M.O. and M.M. acknowledge funding support from STScI GO-1129.01-A and NASA NAO-50-12595. M.O. acknowledges funding support form STScI DDRF D0101.90128. M.M. appreciates support from the Harvard-Smithsonian Center for Astrophysics during this work. This work is in part based on one of the authors' (J.F.) dissertation submitted to University College London, in partial fulfillment of the requirement for the doctorate. This work is in part based on HST archive data 
downloaded from the Canadian Astronomy Data Centre. We thank the support of David Riebel, Lynn Carlson, Brian Ferguson, and NOAO operators for WHIRC observations of these SNe.

\section{REFERENCES}

Andrews, J. E., Gallagher, J. S., Clayton, G. C., et al. 2010, ApJ, 715, 541 Andrews, J. E., Sugerman, B. E. K., Clayton, G. C., et al. 2011, ApJ, 731, 47 Arbour, R., \& Boles, T. 2008, CBET, 1234, 1

Arnett, W. D., \& Fu, A. 1989, ApJ, 340, 396

Barlow, M. J., Sugerman, B. E. K., Fabbri, J., et al. 2005, ApJ, 627, L113

Belley, J., \& Roy, J.-R. 1992, ApJS, 78, 61

Bertoldi, F., Cox, P., Neri, R., et al. 2003, A\&A, 409, L47

Bohlin, R. C., Savage, B. D., \& Drake, J. F. 1978, ApJ, 224, 132

Botticella, M. T., Pastorello, A., Smartt, S. J., et al. 2009, MNRAS, 398, 1041

Brown, P. J., Holland, S. T., Immler, S., et al. 2009, AJ, 137, 4517

Cardelli, J. A., Clayton, G. C., \& Mathis, J. S. 1989, ApJ, 345, 245

Dwek, E., \& Cherchneff, I. 2011, ApJ, 727, 63

Elmhamdi, A., Danziger, I. J., Chugai, N., et al. 2003, MNRAS, 338, 939

Ercolano, B., Barlow, M. J., \& Storey, P. J. 2005, MNRAS, 362, 1038

Ercolano, B., Barlow, M. J., \& Sugerman, B. E. K. 2007, MNRAS, 375, 753

Fabbri, J., et al. 2011, MNRAS, in press

Fabbri, J. 2011, PhD thesis, University College London

Filippenko, A. V., Li, W. D., \& Modjaz, M. 1999, IAU Circ., 7152, 2

Freedman, W. L., Madore, B. F., Gibson, B. K., et al. 2001, ApJ, 553, 47

Gallagher, J. S., et al. 2010, BAAS, 41, 359

Gallagher, J. S., et al. 2011, ApJ, submitted

Garnavich, P., Jha, S., Kirshner, R., Calkins, M., \& Brown, W. 1999, IAU Circ., 7150,1

Gehrz, R. 1989, in IAU Symp. 135, Interstellar Dust, ed. L. J. Allamandola \& A. G. G. M. Tielens (Dordrecht: Kluwer), 445

Hamuy, M., \& Suntzeff, N. B. 1990, AJ, 99, 1146

Hendry, M. A., Smartt, S. J., Maund, J. R., et al. 2005, MNRAS, 359, 906

Karachentsev, I. D., Sharina, M. E., \& Huchtmeier, W. K. 2000, A\&A, 362, 544

Kotak, R., Meikle, W. P. S., Farrah, D., et al. 2009, ApJ, 704, 306

Kozasa, T., Nozawa, T., Tominaga, N., et al. 2009, in ASP Conf. Ser. 414, Cosmic Dust - Near and Far, ed. T. Henning, E. Grön, \& J. Steinacker (San Francisco, CA: ASP), 43

Li, W., Filippenko, A. V., Van Dyk, S. D., et al. 2002, PASP, 114, 403

Li, W., Van Dyk, S. D., Filippenko, A. V., et al. 2006, ApJ, 641, 1060
Li, W., Van Dyk, S. D., Filippenko, A. V., \& Cuillandre, J.-C. 2005, PASP, 117, 121

Lodders, K. 2003, ApJ, 591, 1220

Maeda, K., Tominaga, N., Umeda, H., Nomoto, K., \& Suzuki, T. 2010, Mem. Soc. Astron. Ital., 81, 151

Maguire, K., di Carlo, E., Smartt, S. J., et al. 2010, MNRAS, 404, 981

Mathis, J. S., Rumpl, W., \& Nordsieck, K. H. 1977, ApJ, 217, 425

Maund, J. R., Smartt, S. J., \& Danziger, I. J. 2005, MNRAS, 364, L33

Maund, J. R., \& Smartt, S. J. 2009, Science, 324, 486

Meixner, M., Smee, S., Doering, R. L., et al. 2010, PASP, 122, 451

Mihos, J. C., \& Bothun, G. D. 1997, ApJ, 481, 741

Pastorello, A., Valenti, S., Zampieri, L., et al. 2009, MNRAS, 394, 2266

Patat, F., Barbon, R., Cappellaro, E., \& Turatto, M. 1994, A\&A, 282, 731

Prieto, J. L., Szczygiel, D. M., Kochanek, C. S., et al. 2010, arXiv:1007.0011

Pozzo, M., Meikle, W. P. S., Rayner, J. T., et al. 2006, MNRAS, 368, 1169

Romaniello, M., Patat, F., Panagia, N., et al. 2005, ApJ, 629, 250

Sahu, D. K., Anupama, G. C., Srividya, S., \& Muneer, S. 2006, MNRAS, 372, 1315

Schlegel, D. J., Finkelbeiner, D. P., \& Davis, M. 1998, ApJ, 500, 525

Smith, N., Li, W., Silverman, J. M., Ganeshalingam, M., \& Filippenko, A. V. 2011, MNRAS, 415, 773

Smartt, S. J., Eldridge, J. J., Crockett, R. M., \& Maund, J. R. 2009, MNRAS, 395, 1409

Smartt, S. J., Maund, J. R., Hendry, M. A., et al. 2004, Science, 303, 499

Sparks, W. B. 1994, ApJ, 433, 19

Sugerman, B., Meixner, M., Fabbri, J., \& Barlow, M. 2004, IAU Circ., 8442, 2

Sugerman, B. E. K., Ercolano, B., Barlow, M. J., et al. 2006, Science, 313, 196

Sugerman, B. E. K. 2005, ApJ, 632, L17

Takáts, K., \& Vinkó, J. 2006, MNRAS, 372, 1735

Tsvetkov, D. Y., Volnova, A. A., Shulga, A. P., et al. 2006, A\&A, 460, 769

Utrobin, V. P., \& Chugai, N. N. 2009, A\&A, 506, 829

Van Dyk, S. D., Li, W., \& Filippenko, A. V. 2003, PASP, 115, 1289

Van Dyk, S. D., Li, W., \& Filippenko, A. V. 2006, PASP, 118, 351

Valiante, R., Schneider, R., Bianchi, S., \& Andersen, A. C. 2009, MNRAS, 397 , 1661

Walker, A. R., \& Suntzeff, N. B. 1991, PASP, 103, 958

Welch, D. L., Clayton, G. C., Campbell, A., et al. 2007, ApJ, 669, 525

Wesson, R., Barlow, M. J., Ercolano, B., et al. 2010, MNRAS, 403, 474

Wooden, D. H., Rank, D. M., Bregman, J. D., et al. 1993, ApJS, 88, 477

Woosley, S. E., Hartmann, D., \& Pinto, P. A. 1989, ApJ, 346, 395

Zubko, V. G., Mennella, V., Colangeli, L., \& Bussoletti, E. 1996, MNRAS, 282, 1321 